\title{
Counting Incompossibles*
}

\author{
Peter Fritz and Jeremy Goodman \\ Final Draft.
}

\begin{abstract}
We often speak as if there are merely possible people - for example, when we make such claims as that most possible people are never going to be born. Yet most metaphysicians deny that anything is both possibly a person and never born. Since our unreflective talk of merely possible people serves to draw non-trivial distinctions, these metaphysicians owe us some paraphrase by which we can draw those distinctions without committing ourselves to there being merely possible people. We show that such paraphrases are unavailable if we limit ourselves to the expressive resources of even highly infinitary first-order modal languages. We then argue that such paraphrases are available in higher-order modal languages only given certain strong assumptions concerning the metaphysics of properties. We then consider alternative paraphrase strategies and argue that none of them are tenable. If talk of merely possible people cannot be paraphrased, then it must be taken at face value, in which case it is necessary what individuals there are. Therefore, if it is contingent what individuals there are, then the demands of paraphrase place tight constraints on the metaphysics of properties: either (i) it is necessary what properties there are, or (ii) necessarily equivalent properties are identical and having properties does not entail even possibly being anything at all.
\end{abstract}

We often find ourselves speaking of things there could have been, as much in everyday life as when doing philosophy. We talk about buildings that could have been built but never will be, and of children who could have been born but never will be. Yet in other moods we are inclined to deny that there are any such things. Surely nothing that could be a building fails to ever be one, and nothing that could be a person fails to ever be one. So our opinions appear inconsistent: We appear committed both to there being and to there not being things that could have been people but never are. Which is it?

Philosophical orthodoxy sides with non-being: Nothing that could be a person fails to ever be one. If this is right, then the distinctions that we cognize and communicate when we unreflectively speak of things which could have been people but never are must be understood in other terms. For such distinctions are clearly intelligible. Consider the opening lines of Richard Dawkins' Unweaving the Rainbow:

We are going to die, and that makes us the lucky ones. Most people are never going to die because they are never going to be born. The

\footnotetext{
*Forthcoming in Mind. The final publication is available at https://academic oup.com/mind/article-abstract/126/504/1063/2966528.
} 
potential people who could have been here in my place but who will in fact never see the light of day outnumber the sand grains of Arabia. Certainly those unborn ghosts include greater poets than Keats, scientists greater than Newton. We know this because the set of possible people allowed by our DNA so massively exceeds the set of actual people. In the teeth of these stupefying odds it is you and I, in our ordinariness, that are here. (Dawkins, 1998, p. 1) ${ }^{1}$

What Dawkins is getting at when he says that most people are never going to be born is clearly not obviously false. Yet it is surely false that most people are never going to be born: Every person has been born. So if we wish to speak perspicuously while communicating what he is communicating, we will have to paraphrase him. His talk of 'potential people' and 'possible people' suggests that we paraphrase him as saying that most possible people are never going to be born. But according to philosophical orthodoxy, this paraphrase is little improvement, since all possible people (i.e., things which could have been people) have been (or will be) people, and so have been (or will be) born. The orthodox position therefore faces a paraphrase challenge: to say what substantive claim Dawkins is making without in so doing saying that there are merely possible people (things which could have been people but never are). The challenge is an urgent one. If it cannot be met, then we will have no choice but to take Dawkins' talk of merely possible people at face value, and thus, as we will argue, accept that it is a necessary matter what things there are. ${ }^{2}$

The aim of this paper is two-fold. The first aim is to formulate the challenge illustrated using Dawkins' quote in a general and precise way; this is done in section 1 . The second aim is to explore strategies for how the challenge might be met. Section 2 considers paraphrase strategies available in first-order modal languages, and argues that even given highly infinitary resources they fail to meet the challenge. In section 3 we show that the challenge can arguably be met by moving to a higher-order modal language, but only given one of two controversial views. The first of these views holds that it is necessary what properties there are. The second holds both that necessarily co-extensive properties are identical and that properties of properties can apply to properties which there could not even possibly be. In section 4 we argue that anyone engaged in the project of paraphrase must accept one of these heterodox views, since all other paraphrase strategies are problematic. Assuming that Dawkins is making a substantive claim which can be stated explicitly, we conclude with

\footnotetext{
${ }^{1}$ Merely possible people also figure prominently in ethical debates about the so-called 'nonidentity' problem. See Parfit (1984, chapter 16), Hare (1988), and Hare (2007).

${ }^{2}$ We have slightly oversimplified the orthodox position, which ought to be compatible with the claim that some future machines will count as people and so be people that are never born. What the orthodox position is committed to is that anything that could be a person is at some time a person. (Our ascription of orthodoxy is based on the sociological observation that Williamson's (2013) view that there actually are things that could have been people yet are never born (e.g., Wittgenstein's possible children) has mostly been met with incredulity.)

One might think that human zygotes that fail to develop are merely possible people, on the grounds that people are identical to human organisms that were zygotes before they were people (and hence people are not essentially people). Those who accept such views should replace our talk of merely possible people with talk of merely possible organisms. Fine (2005b) defends the eccentric view that you would still have been a person had you not been born; we can sidestep this dispute by replacing talk of possible people with talk of possible children, since everyone, including Fine, agrees that you would not have been a child had you never been born.
} 
a trilemma in section 5: Either our challenge cannot be met, in which case we have no choice but to accept that it is a necessary matter what individuals there are, or it can be met but only via one of the higher-order paraphrase strategies, in which case one of the two controversial views mentioned above concerning the metaphysics of properties must be true. Despite its disjunctive nature, this conclusion is striking, since most contemporary metaphysicians reject all three of its disjuncts. An appendix establishes the technical results appealed to in section 2 ; the technical results appealed to in section 3 are proved elsewhere.

\section{The paraphrase challenge}

\subsection{Formulating the challenge}

Dawkins' quote provides one example of the sort of discourse that, according to orthodoxy, cannot be taken at face value, and hence, given that we use it to sensibly convey precise ideas about modal reality, must admit of some sort of paraphrase. But there are a host of similar sentences equally in need of paraphrase if orthodox metaphysics is to be maintained. The challenge, then, is to come up with a systematic paraphrase for all such sentences, rather than ad hoc paraphrases for particular examples. Three aspects of this paraphrase challenge need to be clarified: (1) Which sentences are in need of paraphrase? (2) What does it take for one sentence to count as a paraphrase of a given sentence in need of paraphrase? (3) What counts as a systematic paraphrase strategy?

(1): The expression 'most' used by Dawkins is an instance of a well-studied family of variable-binding operators known as generalized quantifiers, other examples of which include the existential and universal quantifiers familiar from first-order logic, 'there are $n$ things such that ...', 'there are infinitely many things such that ...', and 'there are uncountably many things such that ...'? In logic and formal semantics, generalized quantifiers are taken to express structural constraints on the pattern of satisfaction of the conditions expressed by the formulas whose variables they bind. But in Dawkins' quote and in relevantly similar discourse, generalized quantifiers seem to express structural constraints on the pattern of satisfaction of those conditions by all possible individuals. For the purposes of this paper, we understand the paraphrase challenge to apply to all such modalized uses of generalized quantifiers. In delineating the scope of our challenge in this way, we rely on the one hand on established usage of the term 'generalized quantifier' in linguistics and logic, and on the other hand on paradigmatic examples such as Dawkins' quote as well as further examples given below to settle what we call a 'modalized use' of such a quantifier. ${ }^{4}$

(We should note that, although throughout this paper we will engage in the very modalized quantification that we are exploring strategies for eliminating, such talk will not figure in any of the paraphrases we will be considering

\footnotetext{
${ }^{3}$ We are using 'most' in the logician's sense, on which 'most $F$ s are $G$ s' means that there are more $F$ s that are Gs than Fs that aren't Gs. See Westerståhl (2011) for a concise introduction to generalized quantifiers.

${ }^{4}$ Although we limit the challenge to generalized quantifiers here, this is not because we think that the challenge only arises though generalized quantifiers, but only to give the challenge specific boundaries. There are other paraphrase challenges; for example, the challenge to make sense of modalized plural quantification. Goodman (2016) argues that the paraphrase strategy sketched in section 3.2 does not generalize to the plural case.
} 
and so is not objectionable. We will, however, deliberately refrain from theorizing in terms of 'possibilia' that 'exist' only in 'non-actual possible worlds'. We agree with Williamson (2013, chapter 1 ) that such formulations at best obscure the most productive questions in their vicinity - namely, questions that can be formulated in the language of quantified modal logic, on an unrestricted interpretation of its quantifiers and a metaphysical interpretation of its modalties (see section 1.2). In particular, 'possible worlds'-talk prejudices the issues of higher-order contingency discussed in section 3.3; see Williamson (2013, chapter 6) and Stalnaker (2012, chapter 2).)

(2): For a given sentence $S$ involving a modalized use of a generalized quantifier, we will count a sentence $P$ as a paraphrase of $S$ just in case $P$ expresses the proposition conveyed by $S$ - at least up to a fairly demanding standard of 'metaphysical equivalence', as discussed in section 1.3 (for brevity, this qualification will usually be left tacit). This proposition need not be the proposition (literally) expressed by $S$, at least not on the interpretation of its quantifiers and modalities at issue when orthodox metaphysicians say 'There are no merely possible people'. We will assume that, in many cases, we understand sufficiently well which proposition is being conveyed by a modalized use of a generalized quantifier to make firm judgements about whether a proposed paraphrase expresses that proposition.

(3): Any successful answer to the paraphrase challenge must be systematic, in the sense that it should not require any ingenuity to apply in any specific case. Considering only finitary languages, one could try to make this requirement formally precise using the tools of recursion theory, but since we will discuss languages with expressions of infinite length, we cannot do so here. However, for the purposes of evaluating the strategies to be discussed below, it suffices to informally state that any successful strategy must constitute an algorithm that cares only about the form of the sentences being paraphrased. ${ }^{5}$

The general challenge can now be summed up as follows: ${ }^{6}$

Paraphrase Challenge: Provide a systematic strategy that, for any sentence involving modalized quantification, produces a new sentence, not involving modalized quantification, that expresses the proposition conveyed by the original sentence. ${ }^{7}$

Admittedly, this is all very abstract; a range of further examples illustrating this challenge will be given in section 2 .

\subsection{Context sensitivity}

Both quantifiers and modal adverbs breed context-sensitivity, in that sentences containing them can be used literally to express different propositions on differ-

\footnotetext{
${ }^{5}$ One could strengthen this requirement by demanding that the strategy be compositional, in the sense that the paraphrase of a sentence $\varphi$ must occur as a subformula of the paraphrase of any sentence $\psi$ of which $\varphi$ is subformula. But this demand strike us as unduly restrictive: Russell's celebrated theory of descriptions, after all, fails to be compositional in this sense.

${ }^{6}$ Fine (2003), Leuenberger (2006) and Williamson (2010, 2013) raise similar challenges. Lewis (2004), Sider (2006) and Szabó (2006) raise analogous challenges for tensed quantifiers. But note that the way we conceive of our paraphrase challenge differs substantially from the ways in which most of the aforementioned authors conceive of their respective challenges.

${ }^{7}$ To be clear: it is particular uses of (interpreted) sentences, as opposed to sentence types, that do or don't involve modalized quantification, and such modalization may characterize some but not all occurrences of generalized quantifiers in the relevant sentences.
} 
ent occasions of use. So perhaps we speak truly both when in unreflective moods we utter 'Most possible people are never going to be born' and when in more metaphysically minded moods we utter 'There are no merely possible people'. We therefore stipulate that in the formulation of the paraphrase challenge the context-sensitivity of 'there are' and 'possible' in the paraphrasing sentences should be resolved uniformly with the way in which orthodox metaphysicians resolve it when they utter 'There are no merely possible people' - call this the orthodox interpretation.

Why is it an urgent challenge to provide such a paraphrase? Because it is widely agreed that there is a particular way in which we should uniformly resolve the context-sensitivity of our quantifiers and modal adverbs when we do metaphysics. ${ }^{8}$ It follows that when engaging in metaphysical theorizing we cannot accept both of the above two sentences as literally true. Since orthodox metaphysicians accept 'There are no merely possible people' as literally true, they cannot likewise accept 'Most possible people are never going to be born'. So they must provide some other way of making sense of the claim that we would normally communicate by uttering the latter sentence, and do so holding fixed the meaning of their quantifiers and modal adverbs.

One might also appeal to phenomena such as vagueness or ambiguity to undermine the assumption that the uses of sentences we claim to be in need of paraphrase convey a single proposition to be expressed by a paraphrasing sentence. Alternatively, one might simply deny that these uses succeed in conveying any propositions other than the ones they literally express on the orthodox interpretation. We think that the partial success of various paraphrase strategies to be explored below shows that these views are mistaken. For a wide range of cases of modalized quantification, paraphrase strategies can be found which plausibly satisfy the requirements laid down above. In these cases, not only does it seem that a particular proposition is conveyed, but we seem to be able to express it using an orthodoxly-interpreted sentence that means something different than the original sentence would mean on the same interpretation. Since these cases are relevantly similar to other cases of modalized quantification, we conclude that allowing us to convey such propositions is a general feature of modalized quantification, regardless of the quantifier in question.

(One might think that our paraphrase challenge can be met on the cheap by introducing new quantifier-like expressions whose semantic contribution to the sentences in which they occur is stipulated to make those sentences literally express the propositions that would be conveyed by corresponding modalized uses of the corresponding sentences containing unrestricted quantifiers. But given the present dialectic, the important question is whether such a stipulation could succeed on the assumption that the paraphrase challenge cannot be met in any

\footnotetext{
${ }^{8}$ On quantifiers, see Williamson (2003), Dorr (2005), and Sider (2009); on modal adverbs, see Kripke (1980 [1972]) and Plantinga (1974). For general discussion of such metametaphysical issues, see the papers in Chalmers et al. (2009). The methodological assumption about quantifiers has recently been questioned under the heading of 'ontological pluralism'; see McDaniel (2009) and Turner (2010). Someone sympathetic to ontological pluralism might, in the present setting, suggest that quantifiers come in two fundamental families - 'actualist' and 'possibilist' - such that, for example, $\operatorname{most}_{p}$ things that are possibly people are never going to be born, yet everything $a$ that is possibly a person is at some time born. The strategy rejects the paraphrase challenge by positing an ambiguity: modalized quantification just is possibilist quantification. We won't consider such views here, other than to note that the considerations in footnote 9 tell against the most obvious version of the picture.
} 
other way, and this strike us as highly implausible. ${ }^{9}$ )

\subsection{The standard of paraphrase}

Understanding the paraphrase challenge in terms of propositional identity will likely be rejected by those who accept fine-grained theories of propositions that would render the challenge so construed hopelessly demanding. It would be natural for friends of such views to instead understand the challenge in terms of some relation of 'metaphysical equivalence' between propositions, such as corresponding to the same state of affairs. For example, proponents of structured propositions will distinguish the proposition that Hesperus is a planet from the proposition that Hesperus is a planet and Hesperus is a planet, and Fregeans will distinguish the proposition that Hesperus is a planet from the proposition that Phosphorus is a planet, but everyone ought to recognize a sense in which these propositions make the same demands on reality. All of what follows could be reframed in terms of such a notion, although for brevity we will continue to frame the paraphrase challenge in terms of propositional identity.

One might wonder whether anything interesting can be said about our paraphrase challenge without making substantial assumptions about propositional granularity. In particular, one might wonder whether the challenge becomes trivial on a sufficiently coarse grained theory of propositions. To see that it does not, consider the extensionalist view according to which there are only two propositions: the True and the False. On this view, the propositions conveyed using modalized quantifiers can clearly be expressed in all sorts of other terms. But answering the paraphrase challenge requires a systematic paraphrase, which is only sensitive to the form of the sentences being paraphrased (understood as including facts about which quantifier-occurrences are modalized). And extensionalism suggests no such algorithm. (It is worth noting that certain eccentric metaphysical views would support systematic paraphrases given the assumption that propositions are individuated modally. For example, if one thought that there were only finitely many possible contingent beings, then one should

\footnotetext{
${ }^{9}$ The technical results discussed in section 3.6 also entail that the resulting view would have some pretty unattractive features. There we show that, given natural assumptions, contingency in which properties and propositions there are entails that we cannot use the resources of higher-order modal logic to meet the paraphrase challenge for the quantifier 'there are uncountably many'. Since, as we argue in section 3.2 , the paraphrase challenge can be met using the resources of higher-order modal logic if it is not contingent what properties and propositions there are, and we are presently exploring the prospects of introducing 'primitive' modalized quantifiers on the assumption that the challenge cannot be met in that way, let us consider the prospects of the primitivist strategy on the assumption that the modalized 'there are uncountably many' cannot be defined using the resources of higher-order modal logic. Now observe that modalized existential and universal quantifiers of all orders can be defined in such a setting, using the strategy from section 2.1. So not only will modalized 'there are uncountably many' not be explicable using unrestricted quantifiers and modal operators, but it will also not be explicable using modalized higher-order quantifiers in the way that the unrestricted 'there are uncountably many' is standardly explicated in higher-order terms. This leaves proponents of the primitivist strategy in an awkward position. For example, presumably they will want to accept the validity of 'If modalized-most $F$ s are $G$ s, and modalized-thereare-uncountably-many $F_{\mathrm{s}}$, then modalized-there-are-uncountably-many $G$ s'. But unlike in the case of unrestricted quantification, where the corresponding schema is a theorem of higherorder logic when the quantifiers 'most' and 'there are uncountably many' are replaced with their higher-order definitions, the proponent of primitivist modalized quantifiers would seem to have no account of the validity of the modalized schema, since the modalized quantifiers will not by their lights admit of any corresponding definitions in modalized higher-order terms.
} 
be able to systematically paraphrase all modalized generalized quantifiers in a first-order modal language with infinitary conjunction. But this sort of modal finitism is wildly implausible.)

Finally, it is important to clearly distinguish our paraphrase challenge from a related challenge that might be suggested by our technical appendix and by much of the related literature: namely, to produce a paraphrase that is logically equivalent to the sentence being paraphrased. The idea is to imagine a language with two versions of every quantifier, one 'modalized' and the other 'orthodox', and then try to produce a function that maps every sentence of this language to a logically equivalent sentence not containing any modalized quantifiers. Whether or not this proposal comes apart from ours depends in part on how to understand the contested notion of logical equivalence. The challenges as we have formulated it sidesteps that thorny question.

\subsection{Model theory}

To formulate paraphrase strategies with sufficient generality and precision we need to work with formal languages. Having done so, we can then use possibleworlds model theory to get some evidence regarding the success of a proposed paraphrase strategy. To do so, we formulate both the sentences in need of paraphrase and the sentences that are supposed to paraphrase them in a formal language interpreted over such models. In doing so, it is of course essential that the relevant languages include expressions formalizing the modalized uses of generalized quantifiers occuring in sentences to be paraphrased. We can then see whether, as far as the model theory is concerned, a given sentence in need of paraphrase conveys the proposition expressed by a given sentence offered as its paraphrase, by seeing whether the two sentences are true in the same models. This standard must be applied cautiously, since being true in the same models is not obviously equivalent to conveying the same proposition. But it is at least something of a guide, in a sense that can be made precise as follows.

Call a class of models probative just in case, for any two sentences interpreted over those models, the two sentences convey the same proposition only if they define the same class of those models. ${ }^{10}$ In the case of sentences containing no modalized quantifiers, the proposition conveyed will just be the proposition literally expressed on the orthodox interpretation. From the assumption that a given class of models is probative, we can argue that a proposed paraphrase is inadequate by showing that some sentence in need of paraphrase and its proposed paraphrase define different classes of models: by probativity, it follows that the proposition the former conveys is distinct from the proposition the latter expresses, and so the paraphrase challenge has not been met. More generally, we can argue that a certain sentence has no paraphrase in a given formal language by showing that no sentence of that language defines the same class of models as the sentence in need of paraphrase defines. The model-theoretic results appealed to in sections 2 and 3 are of this form.

\footnotetext{
${ }^{10}$ More precisely: any interpretation of the non-logical constants of the language determines a function from sentences of the language to the propositions conveyed by those sentences, and a class of models is probative for that language just in case, for any interpretation of its non-logical constants, two sentences convey the same proposition on this interpretation only if they define the same class of models, where the class of models defined by a sentence is the class of models in which it is true.
} 
Probative classes of models do not straightforwardly give us a way of arguing that a given paraphrase is adequate. ${ }^{11}$ But we can still use probative classes of models as a heuristic in assessing the adequacy of candidate paraphrases, to be supplemented by independent judgments of propositional granularity. Every probative class of models provides a necessary condition of adequacy on any candidate paraphrase strategy. Satisfying such conditions is non-trivial, and so provides substantial, though defeasible, evidence for a strategy's adequacy.

The classes of models appealed to below are versions of the variable domain possible-worlds models first developed by Kripke, on which it is relatively straightforward to interpret modalized generalized quantification. Some necessary truths, such as the claim that there are at least two possible things and the claim that there are at least three possible things, differ with respect to which such models they are true in. Necessarily equivalent sentences might therefore define different classes of models. Those who think that any necessarily equivalent sentences express the same proposition therefore cannot accept these classes of models as probative. But the relevant classes of models can still be used to give substantial, though defeasible, evidence for a strategy's inadequacy, by suggesting that no systematic paraphrase is available, for the reasons mention in section 1.3 .

\section{$2 \quad$ First-order paraphrases}

The expressive resources of standard first-order modal logic provide the resources to answer the paraphrase challenge in a certain restricted range of cases. To help illustrate our abstract challenge and to delineate precisely how far such solutions go, we will start with some simpler modalized generalized quantifiers. We return to Dawkins' quote at the end of this section. When considering a certain formal language, we will say that the paraphrase challenge can be met for a certain generalized quantifier just in case for every sentence in this language in which the only modalized quantifier occurring in it is the modalization of that quantifier, there is a sentence in this language containing no modalized generalized quantifiers which paraphrases it. For further details, see the appendix, section A.1.

\subsection{A possible knife}

In a knife factory there is a spare handle and a spare blade. No knife will ever be made using either of them. Nevertheless, we might naturally say that there is a possible knife that could have been made from this blade and handle had the two been joined. ${ }^{12}$ But if, in keeping with metaphysical orthodoxy, we wish to deny that there are any such possible knives, then this claim, like Dawkins', is in

\footnotetext{
${ }^{11}$ Such an argument would require an individuating class of models: one such that, on any interpretation of their non-logical constants, any two sentences that define the same class of models convey the same proposition. Assuming our language contains classical Boolean connectives, there could only be such a class of models if the class of conveyable propositions forms a Boolean algebra, a hypothesis we don't want to presuppose.

${ }^{12}$ Where it is clear from context, we will use 'possible $\varphi$ s' as shorthand for 'possible things that are possibly $\varphi$ ' and 'merely possible $\varphi$ s' as shorthand for 'possible things that are possibly $\varphi$ and never $\varphi$ '. On this usage, Saul Kripke is a merely possible circus performer.
} 
need of paraphrase. Luckily, the following paraphrase is available: It is possible for there to be a knife made by joining this handle and this blade.

\subsection{Two possible knives}

In the knife factory there is a spare handle and two spare blades. No knife will ever be made using any of these materials. Nevertheless, we might naturally say that there are two possible knives that could be made by joining the handle with one of the blades.

The most flatfooted generalization of the paraphrase strategy from the previous section will not work: It is not possible for there to be two knives made by putting together these materials, since the handle would have to be part of both knives at once, which is impossible. A different strategy is needed. Luckily, the following paraphrase is available: It is possible for there to be a knife made by joining this handle with one of these blades such that it is possible for there to be a different knife made by joining this handle with one of these blades. ${ }^{13}$

This strategy generalizes to cases with any finite number of spare blades. In general, we can paraphrase the claim that there are $n$ possible knives that could be made from these materials by embedding $n$ occurrences of the phrase 'it is possible for there to be a knife made from these materials' in each other: It is possible for there to be a knife $x_{1}$ made from some of these materials such that it is possible for there to be a knife $x_{2}$ distinct from $x_{1}$ made from some of these materials such that ... it is possible for there to be a knife $x_{n}$ distinct from each of $x_{1} \ldots x_{n-1}$ made from some of these materials. Unlike the flatfooted generalization of the paraphrase strategy from the previous section, we need not assume that it is possible for there to be more than one object that is possibly a knife made from some of these materials. In other words, the strategy is compatible with the pairwise incompossibility of the $n$ merely possible knives. We achieve this compatibility by interleaving $n$ possibility operators and $n$ existential quantifiers.

Notice that the claim that these merely possible knives are pairwise incompossible is stronger than the claim that, necessarily, no two of them are knives. One might think that, necessarily, everything that is possibly a knife is at some time a knife, without thinking that, necessarily, everything that is possibly a knife is a knife. On such a view, it might be possible for there to be all of the merely possible knives after all, were the handle joined with each of the blades one after another. One might think that it is therefore unnecessary that our paraphrase strategy be compatible with pairwise incompossibility. Why not instead use the simpler paraphrase: It is possible for there to be $n$ things each of which is at some time a knife made from some of these materials? The problem is that the proposal doesn't generalize: there are two possible dishes - an omelette and a soufflé - that I could make from these eggs, but it is not possible that at some time I make an omelette from these eggs and at another time I make a soufflé from these same eggs. So we need paraphrases compatible with pairwise incompossibility.

\footnotetext{
${ }^{13}$ Throughout this paper cardinality quantifiers should be understood as claiming that there are at least the relevant cardinality of things. In particular, we are not claiming that there are only two possible knives that could be made from the handle and blades.
} 


\subsection{Infinitely many possible knives}

In Hilbert's knife factory there is a spare handle and infinitely many spare blades. No knife will ever be made using any of these materials. Nevertheless, we might naturally say that there are infinitely many possible knives that could be made by joining the handle with one of the blades. ${ }^{14}$

This claim is no less in need of paraphrase than the claim that there are two possible knives that could be made by joining the handle with one of the blades. Yet unlike the latter claim, this claim cannot be paraphrased in a standard firstorder modal language. This is an instance of a more general limitative result: In standard first-order modal logic enriched with all generalized quantifiers, our paraphrase challenge can be met for only those generalized quantifiers that can be defined in standard first-order logic. ${ }^{15}$ Since 'there are infinitely many ...' cannot be defined in standard first-order logic, it follows that not all modalized claims of the form 'there are infinitely many possible ...' can be expressed in a standard first-order modal language, no matter which (unmodalized) generalized quantifiers the language includes. If we hope to paraphrase such claims, we must move to a language whose expressive resources go beyond those of standard firstorder modal logic.

Luckily, there are languages in which we can paraphrase such claims. If we enrich our first-order modal language with a device for forming infinite conjunctions, we can then paraphrase claims of the form 'there are infinitely many possible ...' as infinite conjunctions of the corresponding claims of the form 'there are $n$ possible ...', for all natural numbers $n$. We see nothing illegitimate about paraphrase strategies that employ conjunctions of arbitrary sets of formulas, or that employ infinitary analogues of standard existential and universal quantifiers capable of binding arbitrary sets of variables. ${ }^{16}$ The important question is whether such strategies are sufficiently general to answer our paraphrase challenge.

\subsection{Uncountably many possible knives}

In Cantor's knife factory there is a spare handle and infinitely many spare blades. No knife will ever be made using any of these materials. Nevertheless, we might naturally say that there are uncountably many possible knives that could be made by joining the handle with one of the blades.

This claim is no less in need of paraphrase than the claim that there are infinitely many possible knives that could be made by joining the handle with one of the blades. Yet unlike the latter claim, this claim cannot be paraphrased in a standard first-order modal language in which we can form conjunctions

\footnotetext{
${ }^{14}$ For those worried about the possibility of infinitely many non-overlapping concrete objects, note that the examples below involving electrons and involving monozygotic twins illustrate the same point concerning infinitary incompossibility without assuming the possibility of an infinity of spare blades.

${ }^{15}$ See Theorem 1 of Appendix A.2. The above conclusion relies on the assumption that the model-theoretic criterion of adequate paraphrases faithfully captures the intended one, as discussed in section 1.4

Throughout we will be considering modal languages that include devices for 'undoing' or 'scoping out of' modal operators. Such devices are well studied and correspond to certain uses of 'actually' and 'in fact' in philosophers' English; for technical details, see Fine (1977a, section 6), Hodes (1984), and Correia (2007).

${ }^{16}$ See Dickmann (1985) for discussion of such languages.
} 
of arbitrary sets of formulas and existential quantifications that bind arbitrary sets of variables, and which also includes all generalized quantifiers. This is an instance of a more general limitative result: In such a language, our paraphrase challenge can be met for only those generalized quantifiers that are definable in first-order logic with infinitary conjunction (but only finitary existential and universal quantification). ${ }^{17}$ Since 'there are uncountably many ...' is not definable in first-order logic with infinitary conjunction but only finitary quantification, it follows that not all modalized claims of the form 'there are uncountably many possible ...' can be expressed in the aforementioned modal language. If we hope to paraphrase such claims, we must move to a yet more expressive language.

This result might come as a surprise to those familiar with infinitary languages, since the unmodalized 'there are uncountably many ...' can be paraphrased in standard infinitary first-order logic as 'some $x_{1}, x_{2}, \ldots$ are pairwise distinct and are each ...', where ' $x_{1}, x_{2}, \ldots$ ' stands for an uncountable set of variables and 'pairwise distinct' and 'are each ...' stand for the corresponding uncountable conjunctions. The key observation is that in our language we cannot bind all uncountably many free variables in ' $x_{1}, x_{2}, \ldots$ are pairwise distinct and are each ...' without binding uncountably many of them at once with a single existential quantifier, forcing them to have the same scope. But as we saw in section 2.2, paraphrasing modalized existential quantification requires all variables so-bound to have different scopes, since for any two of them there must be a possibility operator with intermediate scope to accommodate pairwise incompossibility.

This suggests a new paraphrase strategy. Suppose we move to a language in which infinitary quantification is achieved not by letting existential and universal quantifiers bind infinitely many variables at once, but instead by allowing for strings of infinitely many existential and universal quantifiers. Perhaps in such a language we can paraphrase claims of the form 'there are uncountably many possible ...' as claims of the form 'possibly, for some $x_{1}$, possibly, for some $x_{2}$, etc.: $x_{1}, x_{2}$, etc. are pairwise distinct and are each possibly ...' . Here, 'etc.' indicates an uncountable sequence: in the first case for an uncountable string of interwoven possibility operators and existential quantifiers, and in the second case for an uncountable string of variables. ${ }^{18}$

In Appendix A.3 we give a formal syntactic characterization of quantified modal languages allowing for such infinite embeddings, and provide a modeltheoretic semantics relative to which our paraphrase challenge can indeed be met for 'there are uncountably many possible ...' in the above way. For the sake of argument we will grant that languages allowing for infinitely embedded quantifiers and modal operators are legitimate for the purpose of paraphrasing modalized quantification. ${ }^{19}$ Again, we will focus on the question of whether such languages are sufficiently expressive to answer our paraphrase challenge.

\subsection{Most possible people}

Let us finally return to Dawkins' claim that most possible people will never be born. It turns out that it cannot be paraphrased even in the highly infinitiary

\footnotetext{
${ }^{17}$ See Theorem 2 of Appendix A.2.

${ }^{18}$ Fine (2003, section 4) makes a similar proposal.

${ }^{19}$ Williamson (2013, chapter 7) questions their legitimacy in a related context; Fine (forthcoming) replies.
} 
language considered in the previous section. Again, the inability to paraphrase this sentence follows from a more general result: In a first-order modal language enriched with all generalized quantifiers and in which we can infinitely nest conjunctions, disjunctions, existential and universal quantifiers, and modal operators, our paraphrase challenge can be met for only those generalized quantifiers that are definable in a non-modal first-order language in which we can infinitely nest conjunctions, disjunctions, and existential and universal quantifiers. ${ }^{20}$ Since the unmodalized 'most ... are ...' is not definable in the latter non-modal language, it follows that the modalized 'most possible ... are ...' cannot be paraphrased in the former modal language.

One might hope to answer our paraphrase challenge by moving to an even more infinitary language in which we can form conjunctions of proper classes of formulas and have strings of quantifiers and modal operators that bind proper classes of variables. The general idea would be to embed some enormous conjunction within a string of interleaved possibility operators and existential quantifiers that bind as many variables as there are possible individuals. ${ }^{21}$ But we see no consistent way of implementing this strategy. Presumably, for any formula of any language and any variables that occur in it, there should be some formula of some language such that those variables are all and only the variables that occur in it. It then follows by a plural version of Cantor's diagonal argument that no formula contains as many variables as there are possible individuals, since there would have to be more formulas than possible individuals, which is inconsistent, since formulas are individuals. To be clear: the problem is not that a certain specified formula fails to be an adequate paraphrase but rather that it is impossible for there to be a formula satisfying the given specification. ${ }^{22}$

We conclude that the paraphrase challenge cannot be met in even highly infinitary first-order modal languages. In retrospect, this should be somewhat unsurprising. After all, the way we actually explicate generalized quantifiers like 'there are uncountably many ...' is not using infinite conjunction and quantification, but through set-theoretic or higher-order resources. In the next section we explore the possibility of using such resources to answer our paraphrase challenge.

\footnotetext{
${ }^{20}$ See Theorem 5 of Appendix A.4. This result vindicates the skepticism about the expressivity of such languages voiced in Leuenberger (2006, p. 157) and Williamson (2013, p. 354).

${ }^{21}$ Fine (1977a, section 6) advocates such a proposal.

${ }^{22}$ Fine might resist the diagonal argument by rejecting standard plural logic in favor of a logic akin to the non-standard class theory of Fine (2005a), which allows for a universal class. One might also deny that we should think of formulas as individuals (i.e., as sequences of expressions), but instead treat them as well-orderings of expressions, understood in higherorder terms. But the problem then immediately reoccurs if we think that there is contingency in what conditions (e.g., well-orderings) there are, as we discuss in section 3. Generalized quantifiers and their modalizations make just as much sense concerning well-orderings as they do concerning individuals. But using the present strategy to paraphrase modalized generalized quantification over well-orderings would require as many second-order variables as there are well-orderings, and hence well-orderings of variables, which leads to another cardinality crash.
} 


\section{$3 \quad$ Higher-order paraphrases}

\subsection{Singling out merely possible knives}

Why are we so confident that there are uncountably many possible knives that could be made from the materials in Cantor's knife factory? Because the factory contains uncountably many blades, each of which could, together with the handle, compose a knife were the two joined, and any two of which are such that, necessarily, any knife made with one of them could not possibly be made with the other. In other words, we think that there are uncountably many possible knives that could be made using those materials because we know that there are uncountably many ways of making knives from these materials and that each of these ways would yield a different knife. (Dawkins offers an analogous justification of his claim that most possible people are never going to be born, noting that the possibly exemplified human genetic profiles outnumber all the people there will ever be.)

This suggests a strategy for answering our paraphrase challenge in full generality, including for quantifiers such as 'most'. First, we find some ways of singling out the possible things we are interested in. In the case of the possible knives, for example, we appeal to the conditions under which they would be constructed. We then quantify over these conditions, letting them go proxy for the possible objects themselves. ${ }^{23}$ Of course, we have only worked a simple example involving knives, and even in that case one might reject some of our underlying assumptions. We need to examine more systematically the conditions under which such strategies can be used to paraphrase modalized quantification.

\section{$3.2 \quad$ Haecceities in higher-order logic}

First, we need a more precise statement of what it is for a condition $F$ to single out a possible object $x$, in which case we call $F$ a haecceity of $x$. Say that $F$ is a haecceity of $x$ just in case, necessarily, anything is identical to $x$ just in case it is $F$. It follows, by the principle that what is possibly necessary is necessarily necessary, that, if $F$ is possibly a haecceity of $x$, then $F$ is necessarily a haecceity of $x$, even if $x$ is a contingent being. (Note that it does not follow from the fact that being identical to $x$ is a haecceity of $x$ that being identical to $x$ is the only haecceity of $x$, since we have not assumed that necessarily co-extensive conditions are identical.) Say that $F$ is a haecceity just in case it is possible that there be something of which $F$ is a haecceity. For example, the condition of being such that, actually, you would have been a knife made from handle $h$ and blade $b$ had they been joined to form a knife is a haecceity, since, had $h$ and $b$ been joined to form a knife, there would have been something - that knife of which it was a haecceity. ${ }^{24}$

We can now present the general strategy. The idea is to replace talk of possible $F$ s with talk of haecceities that possibly apply to something that is $F$. For example, instead of saying that there are uncountably many possible knives,

\footnotetext{
${ }^{23}$ Related strategies were independently proposed by Plantinga (1976) and Fine (1977a, section 2).

${ }^{24}$ In the interest of accessibility, we formulate these definitions in natural language. Some readers might find it helpful to state them formally: $F$ being a haecceity of $x$ may be regimented as $\square \forall y(y=x \leftrightarrow F y)$, for which we write $H(F, x) . F$ being a haecceity may be regimented as $\diamond \exists x H(F, x)$, for which we write $H(F)$.
} 
we might say that there are uncountably many haecceities each of which is possibly instantiated by a knife and no two of which are possibly instantiated by the same thing. Parallel paraphrases can be given for all generalized quantifiers. The strategy requires moving to a higher-order modal language in which we can quantify into predicate positions; in what follows talk of conditions, properties, and the like should be understood as shorthand for such quantification. ${ }^{25}$

This paraphrase strategy relies on the following assumption if it is to apply generally:

Haecceitistic Plenitude: For every possible individual there is a condition which is its haecceity. ${ }^{26}$

Yet most philosophers who have considered the issue reject this principle: they deny that there is a haecceity of every possible object, usually invoking merely possible elementary particles as purported counterexamples. ${ }^{27}$ The idea is that, just as there is contingency as regards what elementary particles there are, there is parallel contingency in which haecceities of possible elementary particles there are: Had there not been this electron, there would have been no haecceities of it either. On these grounds metaphysicians usually dismiss attempts to answer the paraphrase challenge that try to use haecceities as surrogates for possible objects. $^{28}$

But such dismissals are too quick, since there are more sophisticated paraphrase strategies that can get by with weaker assumptions. Suppose we assume only that haecceitistic plenitude could be true, i.e.:

Haecceitistic Compossibilism: It is possible that for every possible individual there be a haecceity of it. ${ }^{29}$

We can now paraphrase the claim that there are uncountably many possible $F$ s as the claim that, possibly, there are uncountably many haecceities each of which possibly applies to an $F$ and no two of which possibly apply to the same thing. We can in fact prove that, if haecceitistic compossibilism is true, then there is a general paraphrase strategy that answers our paraphrase challenge ${ }^{30}$ - at least insofar as such questions can be adjudicated using standard model-theoretic methods.

The modified strategy is of interest because incompossible possible objects can have compossible haecceities. For example, our incompossible merely possible knives seem to have compossible haecceities, since we seem to be able to

\footnotetext{
${ }^{25}$ Fritz (unpublished) formalizes this paraphrase strategy. Jager (1982) also takes himself to be formalizing something in the vicinity of Plantinga's informal remarks about haecceities, but his project is quite different from ours: we are offering a strategy for paraphrasing modalized first-order quantification in a higher-order language, whereas Jager takes himself to be offering a more metaphysically hygienic alternative to Kripke's (1963) model theory for first-order modal languages.

For more on the operative interpretation of quantification into predicate position, see Prior (1971) and Williamson (2003).

${ }^{26}$ Writing $\Pi$ for the modalized universal quantifier, this can be formalized as follows: $\Pi x \exists X H(X, x)$. The appeal to $\Pi$ can be eliminated using the paraphrase strategy described in section 2.1; see the appendix, section A.1 for formal details.

${ }^{27}$ See, e.g., Fine (2003) and Stalnaker (2012); Plantinga $(1976,1983)$ is a notable exception. ${ }^{28}$ See, e.g., Fine (1977a, section 4), Fine (1985), and Williamson (2013, sections 6.2 and 7.6)

${ }^{29}$ Formally: $\diamond \Pi x \exists X H(X, x)$.

${ }^{30}$ See Fritz (unpublished).
} 
single out each of them in its absence by appealing to the conditions under which it would be manufactured. In particular, in the imagined cases of Hilbert's and Cantor's knife factories, there is a handle and a blade for each of relevant possible knives. Assuming that for any handle and blade, the condition of being a knife made from them (in the appropriate circumstances) is a haecceity, then all of these haecceities are compossible. And while perhaps merely possible elementary particles (and matter more generally) cannot be singled out in their absence, this is not obviously a problem, since bits of matter also seem not to be incompossible in the manner of other possible enmattered objects like possible knives and possible people. Those who think that it is contingent what objects can be singled out might therefore reasonably hope that haecceitistic compossibilism affords a solution to our paraphrase challenge.

\subsection{Incompossible haecceities}

Unfortunately, such optimism would be misplaced. Anyone who rejects haecceitistic plenitude should also reject haecceitistic compossibilism, both with respect to the haecceities of possible enmattered objects (e.g., possible people) and with respect to the haecceities of possible bits of matter (e.g., possible electrons). We will consider the two cases in turn.

Consider a healthy egg and two healthy sperm neither of which will ever fertilize it. Assume that eggs can be fertilized by at most one sperm and that people have their biological origins essentially. It follows that the possible people who could be born from these biological materials are incompossible. But unlike the case of possible knives, we do not seem to be able to single out the possible people who could be born from these biological materials. This is because, for each sperm, there is more than one possible person who could have had it and the egg as its biological origins, since such biological origins could have produced monozygotic twins. We seem to have no way of singling out any such merely possible person from its merely possible twins. E.g., we can't single out one of them as the possibly first-born, since any of them might have been born before any other. We would be able to single out the individual possible twins were they born, but then we wouldn't be able to single out the merely possible people who could be born from the egg and the unused sperm. So we have a counterexample to haecceitistic compossibilism.

The case of matter is somewhat different since bits of matter might not be pairwise incompossible. E.g., it might be that for any two possible electrons, it is possible for there to be both of them. Nevertheless, it seems impossible that there be every possible elementary particle, since, whatever elementary particles there ever are, it is possible that there be an elementary particle that is not one of them. Assuming that necessarily there are no haecceities of merely possible elementary particles, and that elementary particles are essentially elementary particles, it follows that the possible haecceities of possible elementary particles are incompossible.

Since the purported counterexamples to haecceitistic plenitude are, if genuine, also counterexamples to mere haecceitistic compossibilism, the two claims stand or fall together. 


\subsection{The challenge of incompossible haecceities}

If these arguments against haecceitistic compossibilism are sound, then a new strategy is needed to meet the paraphrase challenge. Unfortunately, there are strong model-theoretic indications that there is no available paraphrase even using the highly expressive resources of an infinitary higher-order modal language. In particular, we can show that in a higher-order modal language enriched with infinitary conjunction and quantification, there are pairs of models that agree on all formulas of the language but disagree on whether there are uncountably many objects in the extension of the open formula ' $x$ is a possible being' ${ }^{31}$ Of course, this result is only as instructive as the background model theory is well motivated. And developing variable-domain model theories for higher-order modal languages is a non-trivial technical project with a number of important choice points. Here we can only report that - apart from one exception, to be discussed in section 3.6 - our models are neutral with respect to these choice points and are in keeping with the best motivated and developed extant theories of contingency in what objects can be singled out. ${ }^{32}$

Here is an intuitive characterization of the models. In one model the domain of all possible individuals is countable while in the other model the domain of all possible individuals is uncountable. In both models every finite subset of possible individuals is the domain of some world, all worlds have finite domains, and no two worlds have the same domain; the actual worlds of both models have the same domain. We generate higher-order domains from the first-order domains in accordance with the idea that the distinctions that there are at a world are exactly those that can be drawn using the materials that there are at that world. These two models clearly differ as regards whether they ought to validate 'there are uncountably many possible beings' on the reading we have been trying to paraphrase. But we can prove that the two models validate exactly the same formulas of our infinitary higher-order modal language. In this formal sense, there is no paraphrase of the modalized 'there are uncountably many possible ...' in this language.

We might interpret these models as follows. Suppose that any finite number of people could be born from a particular sperm and egg, that any finite collection of $n$ possible people who could be born from the sperm and egg could be born together as monozygotic $n$-tuplets, and that it is impossible that infinitely many people be born from the sperm and egg. Perhaps this isn't the most plausible view about what sperms and eggs can do, but it is clearly a view we ought to be able to make sense of. We should also be able to distinguish, on the one hand, the conjunction of this view with the claim that there are only countably many possible people who could be born from the sperm and egg, and, on the other hand, the conjunction of this view with the claim that there are uncountably many possible people who could be born from the sperm and egg. The two hypotheses are naturally modeled by the pair of models described above. The

\footnotetext{
${ }^{31}$ This result is the main theorem of Fritz (unpublished); it can be seen as a generalization of the results of Leuenberger (2006), Williamson (2010) and Fritz (2013) to a higher-order setting. It would be prohibitively difficult to generalize this limitative result to a higher-order language that allows for infinite embeddings, but the considerations of section 2.5 give us every reason to expect that the modalization of 'most' resists paraphrase in such a language.

${ }^{32}$ We develop various model theories for infinitary higher-order modal languages in Fritz and Goodman (forthcoming), drawing on the work of Stalnaker (2012), Williamson (2013, chapter 6), and, especially, Fine (1977b).
} 
inability of our higher-order modal language to distinguish the models indicates that it cannot distinguish these two hypotheses, on the assumption that contingency in which individuals there are gives rise to corresponding contingency in which haecceities there are.

\subsection{Implications of haecceitistic plenitude}

Assume for the moment that the higher-order paraphrase strategy outlined in section 3.2 succeeds. Then haecceitistic compossibilism must be true (section 3.4), in which case so too must haecceitistic plenitude (section 3.3). And presumably haecceitistic plenitude is necessary if true. Hence the haecceitistic paraphrase strategy is successful only if the following principle is true:

Weak Haecceitistic Necessitism: Necessarily everything necessarily has a haecceity. $^{33}$

Together with the controversial assumption that necessarily co-extensive properties are identical, weak haecceitistic necessitism entails the following principle:

Strong Haecceitistic Necessitism: It is necessary what haecceities there are. ${ }^{34}$

But what about those who deny that all necessarily co-extensive properties are identical? Might they resist the move from weak to strong haecceitistic necessitism?

Few have wanted to occupy such a position. For among those who deny that necessarily co-extensive conditions are identical, those who have also wanted to resist strong haecceitistic necessitism have usually been motivated by the thought that (i) necessarily equivalent conditions can be distinct by being about different individuals, (ii) which individuals a condition is about is essential to it, and (iii) for all $x$, necessarily, no $F$ is about $x$ unless something is identical to $x$. For example, the condition of being identical to you and the condition of possibly being your biological father are respectively haecceities of you and of your biological father that are about you, and therefore have contingent being on account of your continent being.

By itself this combination of commitments is perfectly consistent with weak haecceitistic necessitism, since it is consistent with the claim that, necessarily, every individual has a qualitative haecceity with necessary being. ${ }^{35}$ But this claim is widely rejected, since it is equivalent to an implausibly strong version of the identity of indiscernibles, according to which, necessarily, every object has a qualitative property that is necessarily co-extensive with being identical to it. ${ }^{36}$ Ordinary material objects, for example, seem to lack qualitative haecceities. Without qualitative haecceities, weak haecceitistic necessitism entails (given the aforementioned assumptions) that, for every material object $x$, necessarily, there

\footnotetext{
${ }^{33} \square \forall x \square \exists X H(X, x)$

${ }^{34} \square \forall X(H(X) \rightarrow \square \exists Y X=Y)$. Note that the identity connective flanked by variables in predicate position can be eliminated in terms of a statement of higher-order indiscernibility; i.e., instead of $X=Y$, we might write: $\forall Z(Z X \leftrightarrow Z Y)$.

Strong haecceitistic necessitism entails weak haecceitistic necessitism given the assumption that necessarily everything has a haecceity, which should be uncontroversial given the operative abundant understanding of conditions; see Williamson (2013, chapter 6).

${ }^{35}$ Taking the notion of a condition being about an individual as primitive, we can define a condition to be qualitative just in case it is not possibly about any individual.

${ }^{36}$ See McMichael (1983) for discussion.
} 
are some individuals that single out $x$, where some individuals single out $x$ just in case there is a haecceity of $x$ which is about all and only them. And this claim is also thought to be wildly implausible, presumably on the grounds that, e.g., had spacetime been empty but for a lone elementary particle, there would not have been any individuals that singled you out. ${ }^{37}$

So it seems that the package of commitments that normally motivates hyperintensional contingentist higher-order metaphysics, and thereby opens up logical space for accepting weak but not strong haecceitistic necessitism, supports an independent argument against weak haecceitistic necessitism, rendering such a split decision ultimately unsustainable. (Furthermore, given that haecceities are generally considered the best candidates for contingent higher-order beings, it would be ad hoc having accepted strong haecceitistic necessitism to not accept necessary being at higher-orders across the board.)

But this argument is too quick. There are certain combinations of metaphysical views which motivate endorsing weak but rejecting strong haecceitistic necessitism. One such view arises from mereological considerations. Say that two material objects coincide just in case they share parts with exactly the same things. It is widely believed that distinct material objects can coincide - for example, a statue and the clay that composes it. According to one widely discussed version of this view, coincidence is ubiquitous in the following sense: for any function from possibilities to possible objects that would have been material objects had the relevant possibility been realized, there is a possible material object whose modal profile of mereological coincidence is given by that function. ${ }^{38}$ It follows that, in any possible one-particle universe, there is an object coincident with the particle in that situation, coincident with you in all possible situations in which you exist, and in all other possible situations fails to be a material object. We might then conjecture that, in a one-particle universe, you could be singled out in terms of your possible mereological relations to such objects. With certain additional assumption, this can be turned into a argument for weak haecceitistic necessitism. ${ }^{39}$ Thus, combining a plenitudinous modal mereology with a hyper-intensional metaphysics of properties would allow one to accept weak haecceitistic compossibilism while rejecting strong haecceitistic compossibilism.

Where would such a split decision leave the haecceitistic paraphrase strategy? The model-theoretic results mentioned give us strong reasons to think that the propositions conveyed by sentences in need of paraphrase and the propositions expressed by their paraphrases would be necessarily equivalent. But given that the envisaged position denies that necessarily co-extensive properties are thereby identical, this gives us little reason to think that the relevant propositions are identical. Defenders of positions like these would need a conception of the granularity of properties and propositions which is fine enough to accept weak haecceitistic necessitism but reject strong haecceitistic necessitism while also being coarse enough to allow for the haecceitistic paraphrase strategy to

\footnotetext{
${ }^{37}$ Fine $(1985$, p. 190) gives an inchoate version of this argument, writing that 'even though a property [of being identical to some merely possible individual] has no [...] counterpart [...] involving actual individuals alone, there may, in each world, exist individuals that suffice to specify its application conditions. This, though, would be a kind of modal freak.'

${ }^{38}$ A closely related thesis is defended by Hawthorne (2006) under the heading of 'plenitude' and is formally explored by Hovda (2013) and Goodman (unpublished).

${ }^{39}$ See Hovda (2013).
} 
succeed. We are skeptical that there is any plausible way of threading this needle. We thus conclude that strong haecceitistic necessitism is a requirement for the success of the haecceitistic paraphrase strategy. Since haecceities are the best candidates for properties with contingent being, we can strengthen this conclusion: the haecceitistic paraphrase strategy succeeds only if it is a necessary matter what properties there are.

\subsection{Paraphrase without compossible haecceities}

As we alluded to earlier, the formal results cited in section 3.4 have a caveat. We will first explain the caveat, and then explain how it suggests a different (non-haecceitistsic) way of using higher-order resources to paraphrase modalized quantification. Although this new strategy requires a combination of commitments that, to our knowledge, no actual philosopher holds, it is neverthless of great interest because, unlike the haecceitistic paraphrase strategy, its success does not require that it be necessary what properties there are.

When developing variable-domain model theories for first-order modal languages, one faces a choice concerning what intensions (functions from worlds to extensions) are admissible values of monadic predicates. In a negative semantics, the intensions must map every world to a subset of the domain of that world. In a positive semantics, intensions can map worlds to any subset of the 'outer domain' of the model (i.e., the union of all domains of all worlds in the model). This distinction between negative and positive semantics carries over straightforwardly to variable-domain model theories for higher-order modal languages, and the theorem mentioned earlier is robust with respect to this decision point.

But a third choice is possible in a higher-order setting. This is because, relative to an assignment of values in the outer domain of a model to free variables in a formula, that formula might express a proposition - i.e., determine a function from worlds to truth values - that is impossible, in the sense of not being in the propositional domain of any world in the model. For example, the intersection (i.e., conjunction) of two incompossible propositions in the domains of different worlds might not be in the domain of any world. But we can still, in a sense, talk about such propositions using modalized existential quantification over their conjuncts (which we can paraphrase in the way described in section 2.1). This then raises the question: should we allow variables in sentential-operator position to have such impossible propositions in their extensions? An affirmative answer is suggested by a higher-order analogue of a thought that is usually used to motivate first-order positive semantics - namely, the thought that there is such a property as self-identity and even contingent non-beings are self-identical. A higher-order analogue of this thought is that there is such a property of propositions as not being the case and (on pain of failing to validate the modal closure of a theorem of propositional logic) for any two possible propositions, either their conjunction is not the case or the conjunction of their negations is not the case. ${ }^{40}$ Model theoretically, this will require allowing variables to have values intensionally equivalent to negation, in which case their extensions will have to

\footnotetext{
${ }^{40}$ An odd feature of higher-order negative semantics, as developed in Fritz and Goodman (forthcoming) based on ideas of Stalnaker (2012), is that it validates a non-classical theory of first-order identity and the claim that there is such a property as self-identity, but in the higher-order case keeps a classical theory of negation and hence invalidates the claim that there is any such property of propositions as negation.
} 
be defined on the aforementioned impossible conjunctions.

On the natural way of developing this kind of 'super-positive' semantics, it will be easy to cook up a translation schema that has the effect of making the bound variables of any type 'range over' the 'super-outer' domain of the model - i.e., over all intensions definable in the model irrespective of whether they (or any lower-order intensions to which they apply at any world) are in the domain of any world of the model. Roughly, 'super-outer-all properties are $\varphi$ ' is translated as the claim that the property of properties being actually such that $\varphi$ is identical to the property of properties being actually co-extensive with oneself. What goes for properties goes for relations, and this is what allows us to solve the problem of incompossible haecceitites: for example, by simulating quantification over impossible relations among incompossible individuals in terms of which we can assess those individals' cardinality. In fact, it can be shown that, relative to a natural class of super-positive higher-order structures, we can paraphrase all modalized quantifiers (in the same sense as the theorem concerning positive and negative semantics assuming haecceitistic compossibilism). Note that, in addition to requiring a super-positive semantics for higher-order predication, the strategy also requires a positive semantics for first-order predication: for example, it requires that I would have had properties (such as self-identity) even had there been no such thing as me. ${ }^{41}$

Notice, however, that the paraphrase strategy depends on higher-order identities - e.g., between the two properties in italics above - that will not generally be accepted by those with hyperintensional theories of properties. This is in contrast with the haecceitistic paraphrase strategy, which did not assume an intensional theory of property granularity. Although the intensional models of course validate that level of granularity, in this respect they can be treated instrumentally as modeling an intensional shadow of a potentially hyperintensional hierarchy, in a sense that can be made formally precise. ${ }^{42}$ But the same cannot be said of the 'super-positive' paraphrase strategy, since in that setting higher-order identity cannot be treated as shorthand for some kind of modally definable equivalence relation - the strategy requires taking identity as primitive, in the sense that it is not definable in the identity-free fragment relative to the super-positive semantics.

We therefore conclude that a successful higher-order paraphrase strategy is available without the assumption of the necessity of what properties there are, but only on the assumption of two contentious principle: first, that properties can apply to propositions and properties which there could not even possibly be, and second, that necessarily equivalent propositions and properties are identical. $^{43}$

\footnotetext{
41 The technical details would take us too far afield and will have to wait for another occasion. ${ }^{42}$ See the discussion of 'hereditary intensionality' in Fritz and Goodman (forthcoming).

${ }^{43}$ One worry for this paraphrase strategy is that it is not clear that an intensional theory of higher-order granularity is compatible the aboutness-theoretic ideas which are usually used to motivate contingency in what properties there are; see Fine (1977b, p. 179-82). In future work we plan to test the robustness of this paraphrase strategy by considering how it generalizes to certain natural hyperintensional but fairly coarse-grained aboutness-theoretic models of higher-order contingency; we are not optimistic about its prospects.
} 


\section{Other expressive resources}

We have seen that there are general strategies for answering our paraphrase challenge using higher-order resources, but that they rely on highly controversial assumptions. Those who wish to reject those assumptions must therefore find other expressive resources with which to answer the challenge, beyond those available in infinitary higher-order modal logic. In this section we argue that it is unlikely that any strategy along these lines can be made to work.

One style of paraphrase that goes beyond the expressive resources of higherorder modal logic is fictionalism. The idea would be to paraphrase the claim that there are uncountably many possible knives that could be made from materials in Cantor's knife factory by saying that, according to a certain fiction, there are uncountably many individuals satisfying a corresponding condition. A full account must of course spell out what the fiction is, what the corresponding condition is, and how the strategy generalizes to cover all modalized uses of generalized quantifiers. A closely related idea is to exploit counterfactuals rather than the notion of truth in fiction: for example, by paraphrasing the aforementioned sentence with the counterfactual claim that, had a certain claim been true (say, had it been necessary what individuals there are), then there would have been uncountably many individuals satisfying a corresponding condition (say, being possibly knives made from the relevant materials). ${ }^{44}$

The most promising versions of these proposals are all instances of a more general paraphrase strategy, appealing to some notion of possibility distinct from metaphysical possibility (fictional possibility, counterfactual possibility, etc.) according to which it is possible for there to be all actually metaphysically possible individuals. We first present this abstract strategy, and then ask what is required of a notion of possibility to serve in this strategy, considering in particular such notions formulated in terms of fictions and counterfactuals. We then argue that even if there is such a notion of possibility and the general strategy can be carried out, this modality gives rise to its own paraphrase challenge, which cannot be solved in the same way.

\subsection{The hyper-possibility paraphrase}

We saw in section 2.2 that incompossibility is the barrier to paraphrasing modalized quantifiers in terms of ordinary quantifiers and modal operators. If we could only somehow bring together all possible individuals in a way that would let us talk about the actual distribution of properties and relations among them, that would be enough. In other words, we could answer the paraphrase challenge if only there were some notion of possibility - call it hyper-possibility - such that it is hyper-possible that there be all actually metaphysically possible individuals, and such that we can use actuality operators to 'scope out' of hyper-possibility operators and thereby, within the scope of the hyper-possible, draw distinctions

\footnotetext{
${ }^{44}$ See Fine (2003, section 5) for a discussion of fictionalist paraphrases, and Fine (forthcoming) for an endorsement and formal development of a variant using an 'according to the supposition' operator; important precursors include Rosen (1990) and Sider (2002). The counterfactual approach is inspired by Dorr (2005, section 3) and Dorr (2008, section 2); see also Woodward (2012). Note that fictionalist paraphrase of modalized quantification should not be confused with fictionalism as a theory about the metaphysics of 'possible worlds', defended by Rosen (1990).
} 
among all actually metaphysically possible things with respect to their actual properties and relations.

Let us illustrate how to carry out this strategy in more detail using the example of Dawkins' claim that most possible people are never born. This requires scope-indicating devices of the sort mentioned in footnote 15. These are, first, the familiar philosophers' 'actually' operator, and second, a less familiar family of 'in fact ${ }_{i}$ ' operators that have the effect of letting the subformula they embed be evaluated at a wider scope indicated by a co-indexed $\uparrow_{i}$. Given these devices, the claim that it is hyper-possible that there be all actually metaphysically possible individuals can be formulated more perspicuously as follows:

It is hyper-possible that $\uparrow_{1}$ actually metaphysically necessarily everything is in fact ${ }_{1}$ identical to something.

This statement now needs to be continued to say that, in some such 'hyperpossibility', most actually metaphysically possible people are actually never born.

It is hyper-possible that: $\uparrow_{1}$ actually metaphysically necessarily everything is in fact ${ }_{1}$ identical to something, and most things such that actually, it is metaphysically possible that they are people are such that, actually, they are never born. ${ }^{45}$

\subsection{Conditions for success}

We know that any notion of hyper-possibility for which the above paraphrase strategy succeeds must be a weaker notion of possibility than the notion of 'metaphysical' possibility we have been operating with thus far. But hyperpossibility must also be sufficiently metaphysically robust to permit quantifying in and 'scoping out'. The question is whether any notion of possibility meets these two requirements. The answer to this question is not at all clear.

Suppose, for example, that we can make sense of a notion of 'logical possibility' such that, for any formula $\varphi$ of a first-order modal language, $\ulcorner$ it is logically possible that $\varphi\urcorner$ is true just in case $\varphi$ is satisfiable relative to a standard possible world semantics for first-order modal logic. Note first that Leibniz's law fails to hold within the scope of logical possibility, since it is logically possible that Hesperus is distinct from Phosphorus but not logically possible that Hesperus is distinct from Hesperus. Given such opacity, one might worry whether one can unambiguously quantify into the scope of this operator. Second, and more importantly, we cannot use an actuality operator to scope out of 'it is logically possible that ...', since, although there are no flying pigs, it is logically possible that there are actually flying pigs. So this notion of logical possibility is not metaphysically robust, and is therefore not suitable to play the role of hyper-possibility.

Further candidates for hyper-possibility can be obtained using the fictionalist and counterfactual resources mentioned above. Hyper-possibility may therefore

\footnotetext{
${ }^{45}$ In a first-order modal language including a hyper-possibility operator $\langle h\rangle$, an actually operator @, the operator $\uparrow_{i}$, an operator $\downarrow_{i}$ formalizing 'in fact ${ }_{i}$ ', as well as the generalized quantifier $\mathcal{M}$ formalizing 'most', this can be written as follows:

$$
\langle h\rangle\left(\uparrow_{1} @ \square \forall x \downarrow_{1} \exists y(x=y) \wedge \mathcal{M} x y(@ \diamond P x, @ \neg B y)\right)
$$
}


be thought of as expressed by the phrase 'according to the fiction that there are all actually possible things ...' or the phrase 'had it been the case that there are all actually possible things, it would have been the case that ...' It is far from clear that these clauses interact as required with quantifiers and scope indicating devices such as actuality operators. But it turns out that we can sidestep that question for present purposes, as the proposed paraphrase strategies suffer from additional problems.

\subsection{Revenge}

The first problem is that it is unclear whether any metaphysically robust notion of hyper-possibility is weak enough to admit the possibility of there being all actually metaphysically possible individuals. Even if some such modality can be used to evade essentialism-induced incompossibility, there is no reason to think it will evade the second sort of incompossibility discussed in section 2.3 - namely, the incompossibility that results from the fact that, necessarily, whatever possible elementary particles there are, it is possible that there be all of them and one more possible elementary particle. The same considerations that lead us to think that all metaphysically possible elementary particles are metaphysically incompossible should lead us to think that all metaphysically possible elementary particles are hyper-incompossible - namely, that it is hypernecessary that, whatever actually metaphysically possible elementary particles there are, it is hyper-possible that there be all of them and one more actually metaphysically possible elementary particle. Compare: it is metaphysically necessary that whatever actually nomologically possible elementary particles there are, it is metaphysically possible that there be all of them and one more actually nomologically possible elementary particle.

We therefore see no reason to think that there is any metaphysically robust notion of hyper-possibility according to which it is hyper-possible that there be all actually metaphysically possible elementary particles. And even if there were such a notion, it would not allow us to answer the paraphrase challenge in full generality, because we could still run a different analogue of the argument from section 2.3. Presumably it is hyper-necessary that, whatever hyper-possible elementary particles there are, it is hyper-possible that there be all of them and one more. It follows that the hyper-possible elementary particles are hyperincompossible. This raises such questions as: Are most hyper-possible elementary particles metaphysically possible? The proponent of the hyper-possibility paraphrase now faces an paraphrase challenge exactly analogous to the one with which we began. It is no help to appeal to an infinite hierarchy of notions of hyper-possibility, since the disjunction of all such modalities will itself be a notion of hyper-possibility to which the revenge argument applies.

\section{Conclusion}

We have raised a paraphrase challenge for anyone who denies that

(1) It is necessary what individuals there are.

After having surveyed the most promising strategies for answering it, we conclude that only two, both appealing to higher-order resources, have a chance of 
success. And even here, their successes respectively require the following highly contentious assumptions:

(2) It is necessary what properties there are.

(3) Necessarily co-extensive properties are identical, properties of individuals can apply to merely possible individuals, and properties of properties can apply to properties which there could even not possibly be.

We conclude that at least one of (1), (2) or (3) is true. ${ }^{46}$

\section{References}

Carlos Areces and Balder ten Cate. Hybrid logics. In Patrick Blackburn, Johan van Benthem, and Frank Wolter, editors, Handbook of Modal Logic, pages 821-868. Amsterdam: Elsevier, 2007.

David J. Chalmers, David Manley, and Ryan Wasserman. Metametaphysics. Oxford: Oxford University Press, 2009.

Fabrice Correia. Modality, quantification, and many Vlach-operators. Journal of Philosophical Logic, 36:473-488, 2007.

Richard Dawkins. Unweaving the Rainbow: Science, Delusion and the Appetite for Wonder. London: Penguin Books, 1998.

M. A. Dickmann. Larger infinitary languages. In J. Barwise and S. Feferman, editors, Model-Theoretic Logics, pages 317-363. New York: Springer-Verlag, 1985 .

Cian Dorr. What we disagree about when we disagree about ontology. In Mark Eli Kalderon, editor, Fictionalism in Metaphysics, pages 234-286. Oxford: Oxford University Press, 2005.

Cian Dorr. There are no abstract objects. In Theodore Sider, John Hawthorne, and Dean W. Zimmerman, editors, Contemporary Debates in Metaphysics, pages 32-64. Malden, MA: Blackwell, 2008.

Fredrik Engström. Generalized quantifiers in dependence logic. Journal of Logic, Language and Information, 21:299-324, 2012.

Kit Fine. Postscript to Worlds, Times and Selves (with A. N. Prior). London: Duckworth, 1977a.

Kit Fine. Properties, propositions and sets. Journal of Philosophical Logic, 6: 135-191, 1977b.

\footnotetext{
${ }^{46}$ Thanks to Simona Aimar, Andrew Bacon, Cian Dorr, Kit Fine, John Hawthorne, Øystein Linnebo, Ofra Magidor, Reinhard Muskens, Agustín Rayo, Tim Williamson, and four anonymous referees and two editors of this journal for comments on earlier versions of this paper, and to audiences at the 17th Oxford Philosophy Graduate Conference in 2013, Washington Square Circle at NYU in 2013, a philosophical colloquium at Humboldt University in 2014, and the CSMN Workshop on Modality, Meaning, and Metaphysics at the University of Oslo in 2014. Peter Fritz gratefully acknowledges the support of an AHRC/Scatcherd European Scholarship and the German Academic Exchange Service. Jeremy Goodman gratefully acknowledges the support of the Clarendon Fund.
} 
Kit Fine. Plantinga on the reduction of possibilist discourse. In J. Tomberlin and Peter van Inwagen, editors, Alvin Plantinga, pages 145-86. D. Reidel, 1985 .

Kit Fine. The problem of possibilia. In Michael J. Loux and Dean W. Zimmerman, editors, The Oxford Handbook of Metaphysics, pages 161-179. Oxford: Oxford University Press, 2003.

Kit Fine. Class and membership. The Journal of Philosophy, 102:547-572, 2005a.

Kit Fine. Necessity and non-existence. In Modality and Tense, pages 321-54. Oxford: Oxford University Press, 2005b.

Kit Fine. Williamson on Fine on Prior on the reduction of possibilist discourse. In Juhani Yli-Vakkuri and Mark McCullagh, editors, Williamson on Modality, Canadian Journal of Philosophy, special issue. forthcoming.

Peter Fritz. Modal ontology and generalized quantifiers. Journal of Philosophical Logic, 42:643-678, 2013.

Peter Fritz. Higher-order contingentism, part 3: Expressive limitations. unpublished.

Peter Fritz and Jeremy Goodman. Higher-order contingentism, part 1: Closure and generation. Journal of Philosophical Logic, forthcoming.

Jeremy Goodman. The Case for Necessitism. DPhil thesis, University of Oxford, 2016.

Jeremy Goodman. Matter and mereology. unpublished.

Caspar Hare. Voices from another world: Must we respect the interests of people who do not, and will never, exist? Ethics, 117:498-523, 2007.

R. M. Hare. Possible people. Bioethics, 2:279-93, 1988.

John Hawthorne. Metaphysical Essays. Oxford: Oxford University Press, 2006.

Jaakko Hintikka and Veikko Rantala. A new approach to infinitary languages. Annals of Mathematical Logic, 10:95-115, 1976.

Harold Hodes. On modal logics which enrich first-order S5. Journal of Philosophical Logic, 13:423-454, 1984.

Paul Hovda. Tensed mereology. Journal of Philosophical Logic, 42:241-83, 2013.

Thomas Jager. An actualistic semantics for quantified modal logic. Notre Dame Journal of Formal Logic, 23:335-349, 1982.

Carol R. Karp. Finite-quantifier equivalence. In J. W. Addison, Leon Henkin, and Alfred Tarski, editors, The Theory of Models: Proceedings of the 1963 International Symposium at Berkeley, pages 407-412. Amsterdam: NorthHolland Publishing Company, 1965. 
Maaret Karttunen. Infinitary languages $N_{\infty \lambda}$ and generalized partial isomorphisms. In Jaakko Hintikka, Ilkka Niiniluoto, and Esa Saarinen, editors, Essays on Mathematical and Philosophical Logic, pages 153-168. Dordrecht: D. Reidel, 1979.

Maaret Karttunen. Model theoretic results for infinitely deep languages. Studia Logica, 42:223-241, 1983.

Ph. G. Kolaitis. Game quantification. In J. Barwise and S. Feferman, editors, Model-Theoretic Logics, pages 365-421. New York: Springer-Verlag, 1985.

Saul A. Kripke. Semantical considerations on modal logic. Acta Philosophica Fennica, 16:83-94, 1963.

Saul A. Kripke. Naming and Necessity. Cambridge, MA: Harvard University Press, 1980 [1972]. First published in Semantics of Natural Language, edited by Donald Davidson and Gilbert Harman, pages 253-355, 763-769, Dordrecht: D. Reidel, 1972.

Stephan Leuenberger. A new problem of descriptive power. The Journal of Philosophy, 103:145-162, 2006.

David Lewis. Tensed quantifiers. In Dean W. Zimmerman, editor, Oxford Studies in Metaphysics, volume 1, pages 3-14. Oxford: Oxford University Press, 2004.

Kris McDaniel. Ways of being. In David J. Chalmers, David Manley, and Ryan Wasserman, editors, Metametaphysics, pages 290-319. Oxford: Oxford University Press, 2009.

Alan McMichael. A problem for actualism about possible worlds. Philosophical Review, 92:49-66, 1983.

Juha Oikkonen. A generalization of the infinitely deep languages of Hintikka and Rantala. In Esa Saarinen, Risto Hilpinen, Ilkka Niiniluoto, and Merrill Provence Hintikka, editors, Essays in Honour of Jaakko Hintikka, pages 101-112. Dordrecht: D. Reidel, 1979.

Derek Parfit. Reasons and Persons. Oxford: Oxford University Press, 1984.

Ahti-Veikko Pietarinen. Semantic games and generalised quantifiers. In AhtiVeikko Pietarinen, editor, Game Theory and Linguistic Meaning, pages 183206. Oxford: Elsevier, 2007.

Alvin Plantinga. The Nature of Necessity. Oxford: Oxford University Press, 1974.

Alvin Plantinga. Actualism and possible worlds. Theoria, 42:139-160, 1976.

Alvin Plantinga. On existentialism. Philosophical Studies, 44:1-20, 1983.

A. N. Prior. Platonism and quantification. In Objects of thought, chapter 3, pages 31-47. Oxford: Oxford University Press, 1971.

Veikko Rantala. Game-theoretical semantics and back-and-forth. In Jaakko Hintikka, Ilkka Niiniluoto, and Esa Saarinen, editors, Essays on Mathematical and Philosophical Logic, pages 119-151. Dordrecht: D. Reidel, 1979. 
Gideon Rosen. Modal fictionalism. Mind, 99:327-354, 1990.

Theodore Sider. The ersatz pluriverse. The Journal of Philosophy, 99:279-315, 2002.

Theodore Sider. Quantifiers and temporal ontology. Mind, 115:75-97, 2006.

Theodore Sider. Ontological realism. In David Chalmers, David Manley, and Ryan Wasserman, editors, Metametaphysics, pages 384-423. Oxford: Oxford University Press, 2009.

Robert Stalnaker. Mere Possibilities. Princeton: Princeton University Press, 2012.

Zoltán Gendler Szabó. Counting across times. Philosophical Perspectives, 20: 399-426, 2006.

Jason Turner. Ontological pluralism. The Journal of Philosophy, 107:5-34, 2010.

Jouko Väänänen. Barwise: Abstract model theory and generalized quantifiers. The Bulletin of Symbolic Logic, 10:37-53, 2004.

Jouko Väänänen. Models and Games. Cambridge: Cambridge University Press, 2011.

Dag Westerståhl. Generalized quantifiers. In Edward N. Zalta, editor, Stanford Encyclopedia of Philosophy. Summer 2011 edition, 2011.

Timothy Williamson. Everything. Philosophical Perspectives, 17:415-465, 2003.

Timothy Williamson. Necessitism, contingentism, and plural quantification. Mind, 119:657-748, 2010.

Timothy Williamson. Modal Logic as Metaphysics. Oxford: Oxford University Press, 2013.

Richard Woodward. Fictionalism and incompleteness. Noûs, 46:781-90, 2012.

\section{A Appendix: Definability of modalized general- ized quantifiers in infinitary first-order modal logics}

In this appendix, we prove and discuss the results on the definability of modalizations of generalized quantifiers appealed to in section 2. In the interest of brevity and readability, we will keep the definitions somewhat informal, and only sketch proofs of the results. ${ }^{47}$

\footnotetext{
${ }^{47}$ The appendix is the work of the first author.
} 


\section{A.1 Well-founded languages}

We start with the more standard languages appealed to in sections 2.3 and 2.4, which require the subformula relation to be well-founded. For simplicity, we assume that we are working in a relational signature, so we don't allow individual constants or function symbols. As usual, we let $\mathcal{L}_{\omega \omega}$ be the language of first-order logic, i.e., the set of formulas built out of atomic predications (including identity statements, = being treated as a logical connective) using negation $(\neg)$, binary disjunction and conjunction $(\vee$ and $\wedge$ ), and existential and universal quantification $(\exists$ and $\forall)$. Likewise, we use $\mathcal{L}_{\infty \omega}$ for the extension of this language obtained by allowing the disjunction and conjunction operators to apply to sets of formulas of arbitrary cardinality (writing them $\bigvee$ and $\Lambda$ ), and $\mathcal{L}_{\infty \infty}$ for the further extension obtained by also allowing existential and universal quantification binding sets of variables of arbitrary cardinality. These languages are interpreted as usual over a model $\mathfrak{A}$ given by a set $|\mathfrak{A}|$ (the domain of quantification) and a relation $R^{\mathfrak{A}}$ of the appropriate arity on $|\mathfrak{A}|$ for each relational symbol $R$ in our signature. We write $\mathfrak{A}, a \vDash \varphi$ for $\varphi$ being true in $\mathfrak{A}$ relative to an assignment function $a$; note that $a$ may be partial, as long as it is defined on all free variables in $\varphi$.

From $\mathcal{L}_{\omega \omega}$ and $\mathcal{L}_{\infty \infty}$, we derive first-order modal languages $\mathcal{L}_{\omega \omega}^{\mathrm{mq}}$ and $\mathcal{L}_{\infty \infty}^{\mathrm{mq}}$ by adding four resources: (i) all generalized quantifiers; (ii) a new kind of variables, called world-variables, which can be used as atomic formulas; (iii) existential and universal quantifiers binding a single world variable; and (iv) an additional operator @ operating on a world variable and a formula. We will mark the difference between the two kinds of variables by using Roman letters for individual variables and Greek letters for world variables.

We interpret the modal languages over the usual Kripke models, understood as tuples $\langle W, D, i, w\rangle$, where $W$ is a set (the 'set of worlds'), $D$ is a function mapping each $v \in W$ to a set $D_{v}$ (the 'domain of $w^{\prime}$ '), and $i$ is a function mapping each relation symbol of our signature to a function which maps every world $v$ to a relation of the appropriate arity on $D_{v}$. We evaluate formulas relative to a world $v$ and an assignment function $a$, writing $\mathfrak{M}, v, a \vDash \varphi$ for $\varphi$ being true in $\mathfrak{M}$ and $v$ relative to $a$. Assignment functions may again be partial, and in addition to mapping individual variables to individuals, now also map world variables to worlds. We let $D_{v}$ be the domain of individual quantifiers - including generalized quantifiers - evaluated at $v$. A world variable is true in just the world assigned to it; world quantifiers range over the set of worlds; and the effect of @ $\xi$ is to let the world of evaluation be the world assigned to $\xi$.

More precise definitions of such modal languages can be found in Williamson (2013, section 7.9) and Fritz (2013), although both use a kind of generalized actuality operators instead of explicit quantification over worlds. We choose explicit quantification over worlds here since this generalizes more naturally to the case of non-well-founded languages; see Appendix A.5 for further discussion of this difference. For now, we only note that it is straightforward to use the resources available here to define the familiar operators for possibility and necessity as follows, where $\xi$ is some world variable not free in $\varphi$ :

$$
\begin{aligned}
& \diamond \varphi={ }_{\mathrm{df}} \exists \xi @ \xi \varphi \\
& \square \varphi={ }_{\mathrm{df}} \forall \xi @ \xi \varphi
\end{aligned}
$$


For more on quantifiers binding world variables, see the literature on hybrid logic, e.g., Areces and ten Cate (2007). See Westerståhl (2011) for a definition of generalized quantifiers and their interpretation, and Fritz (2013, section 1) for generalized quantifiers in modal logic.

We say that a generalized quantifier $\mathcal{Q}$ is definable in a given language just in case adding it to the language does not increase its expressivity, in the sense that every sentence of the expanded language is equivalent to some sentence in the original language (where two sentences are equivalent just in case they are true in the same models and false in the same models - the non-well-founded languages discussed below introduce gaps in the truth-value assignment, so agreement on truth and falsity have to be imposed separately). A helpful reformulation of this notion uses what we might call the canonical sentence $\gamma_{\mathcal{Q}}$ of a generalized quantifier $\mathcal{Q}$, which is simply the result of applying it to a sequence of atomic formulas using relation symbols of appropriate arities, keeping all variables and relation symbols distinct. We can show that in any non-modal language considered here (including the non-well-founded languages to be introduced below), $\mathcal{Q}$ is definable if and only if the language contains a sentence equivalent to $\gamma_{\mathcal{Q}}$.

For any generalized quantifier $\mathcal{Q}$, define the modalization of $\mathcal{Q}$ to be an operator $\mathcal{Q}^{O}$ which when added to any modal language considered here has the following truth-conditions:

$$
\mathfrak{M}, v, a \vDash \mathcal{Q}^{O} \overline{\bar{x}} \bar{\varphi} \text { iff } \mathcal{Q}_{\bigcup_{u \in W} D_{u}}\left(\varphi_{0}\left(\bar{x}_{0}\right)_{O}^{\mathfrak{M}, v, a}, \ldots, \varphi_{l-1}\left(\bar{x}_{l-1}\right)_{O}^{\mathfrak{M}, v, a}\right)
$$

Here, $\mathfrak{M}=\langle W, D, i, w\rangle$ and $\varphi(\bar{x})_{O}^{\mathfrak{M}, v, a}=\left\{\bar{o} \in\left(\bigcup_{u \in W} D_{u}\right)^{n}: \mathfrak{M}, v, a[\bar{o} / \bar{x}] \vDash \varphi\right\}$. (In general, we indicate a sequence of elements by putting a bar over the relevant term, leaving the length of the sequence to be determined by the context. We also adopt the usual convention of writing $a[o / x]$ for the $x$-variant of the assignment $a$ which maps $x$ to $o$, and extend this to the case of tuples in the obvious way.) Definability of modalized generalized quantifiers is understood as before, except that we take two sentences to be equivalent if they are true/false in the same Kripke models in the same worlds. We also extend the definition of the canonical sentence of a modalized generalized quantifier in the obvious way, but note that in the Kripke models used here, the extension of a relation at a world is confined to the individuals at that world, so it is no longer clear that being able to express the canonical sentence of a modalized generalized quantifier implies being able to define it, although of course the converse still holds.

\section{A.2 Definability in well-founded languages}

Theorem 1. The modalization of a generalized quantifier $\mathcal{Q}$ is definable in $\mathcal{L}_{\omega \omega}^{\mathrm{mq}}$ if and only if $\mathcal{Q}$ is definable in $\mathcal{L}_{\omega \omega}$.

Proof. It is straightforward to adapt the proof of Fritz (2013, p. 656, Theorem 1) to the present setting.

Theorem 2. The modalization of a generalized quantifier $\mathcal{Q}$ is definable in $\mathcal{L}_{\infty \infty}^{\mathrm{mq}}$ if and only if $\mathcal{Q}$ is definable in $\mathcal{L}_{\infty \omega}$.

Proof. If $\mathcal{Q}$ is definable in $\mathcal{L}_{\infty \omega}$ then there is a sentence $\varphi$ of $\mathcal{L}_{\infty \omega}$ equivalent to $\gamma_{\mathcal{Q}}$. Define $\varphi^{O}$ to be the result of replacing quantifiers by complex constructions in $\varphi$, replacing 


\author{
$\exists x \psi$ by $\exists \xi(\xi \wedge \diamond \exists x @ \xi \psi)$, and \\ $\forall x \psi$ by $\exists \xi(\xi \wedge \square \forall x @ \xi \psi)$.
}

For any $\chi$ in $\mathcal{L}_{\infty \infty}^{\mathrm{mq}}$ enriched by $\mathcal{Q}^{O}$, replacing every occurrence of $\mathcal{Q}^{O}$ by $\varphi^{O}$ (in which atomic predications are in turn replaced by the relevant subformulas of $\chi)$ results in a sentence of $\mathcal{L}_{\infty}^{\mathrm{mq}}$ which we can prove to be equivalent to $\chi$.

For the other direction, we adapt the proof of Fritz (2013, p. 664, Proposition 10). Let $\mathcal{Q}$ be a generalized quantifier not definable in $\mathcal{L}_{\infty}$. . Consider any infinite ordinal $\alpha$ and let $\mathcal{L}_{\infty \omega}^{\alpha}$ be the class of $\mathcal{L}_{\infty \omega}$ sentences of quantifier rank up to $\alpha$. As noted in Väänänen $(2004$, p. 46 , Lemma 9$), \mathcal{L}_{\infty \omega}^{\alpha}$ has at most $\beth_{\alpha}$ sentences up to equivalence, so the class of models defined by $\gamma_{\mathcal{Q}}$ is not the union of equivalence classes of the relation of satisfying the same sentences of $\mathcal{L}_{\infty \omega \omega}^{\alpha}$. Hence there are models $\mathfrak{A}$ and $\mathfrak{B}$ which satisfy the same sentences of $\mathcal{L}_{\infty \omega \omega}^{\alpha}$, while $\mathfrak{A}$ but not $\mathfrak{B}$ satisfies $\gamma_{\mathcal{Q}}$. By Karp $(1965$, p. 410, Theorem 1$)$, there is a back-and-forth system of length $\alpha$ relating $\mathfrak{A}$ and $\mathfrak{B}$. As in Fritz (2013, p. 664, Lemma 9), we can extend this to a kind of back-and-forth system which holds between Kripke models $\mathfrak{A}^{n}$ and $\mathfrak{B}^{n}$ based on $\mathfrak{A}$ and $\mathfrak{B}$, and conclude from this that $\mathfrak{A}^{n}$ and $\mathfrak{B}^{n}$ satisfy the same sentences of $\mathcal{L}_{\infty}^{\text {mq }}$ up to modal depth $\alpha$ (which can now be understood as world quantifier depth). From the fact that $\mathfrak{A}$ but not $\mathfrak{B}$ satisfies $\gamma_{\mathcal{Q}}$, we can conclude that $\mathfrak{A}^{n}$ but not $\mathfrak{B}^{n}$ satisfies $\gamma_{\mathcal{Q}^{o}}$. Since every sentence of $\mathcal{L}_{\infty \infty}^{\mathrm{mq}}$ has some ordinal modal depth, we conclude that no such sentence defines $\mathcal{Q}^{\infty}$.

\title{
A.3 Non-well-founded languages
}

We now turn to the languages appealed to in section 2.5, which allow for infinitely deep nestings of operators. The literature on such languages goes back to Hintikka and Rantala (1976), whose approach we follow here. According to it, formulas are given by labeled trees - allowing infinite branching as well as infinitely long branches - in which leaf nodes are labeled by atomic formulas and non-leaf nodes are labeled by operators. Here, we will define these languages in a somewhat unorthodox way, in a sense combining the traditional recursive definition of formulas with Hintikka and Rantala's tree-based approach. We motivate setting up the languages in this way at the end of this section; now, we start by defining the syntax and semantics.

Define a tree to be a partial order $\langle T, \leq\rangle$ such that for all $t, t^{\prime} \in T$ :

(a) $\{s: s \leq t\}$ is well-ordered by $\leq$,

(b) there is a leaf (maximal element) $s$ such that $t \leq s$, and

(c) if $\{s: s \leq t\}=\left\{s: s \leq t^{\prime}\right\}$ and the order type of this set is a limit ordinal, $t=t^{\prime}$.

With this, we define the formulas of $\mathcal{N}_{\infty \infty}$ using the standard method of (finitary) structural recursion, but with the following (partly non-standard) clauses:

- For any relation symbol $R$ and variables $\bar{x}, R \bar{x}$ is a formula.

- If $\varphi$ is a formula, then $\neg \varphi$ is a formula.

- $\langle T, \leq, g\rangle$ is a formula, given that 
$-\langle T, \leq\rangle$ is a tree (as defined above),

- $g$ is a function on $T$ mapping any leaf to a formula and any non-leaf to $\vee, \wedge, \exists x$ or $\forall x$ (for some individual variable $x$ ), and

- if $t$ is not a leaf and $g(t) \notin\{\wedge, \vee\}$, then $t$ has a unique successor.

To interpret formulas of $\mathcal{N}_{\infty \infty}$, we adapt Hintikka and Rantala's gametheoretic semantics. Since we have a hybrid syntax, we also proceed with a hybrid semantics, using games only for the step of trees in the usual recursion of truth-conditions relative to a model and an assignment function. As before, we allow assignment functions to be partial, and in this case, don't even require them to be defined on all free variables. As infinite embeddings may introduce truth-value gaps, we define separate properties of truth and falsity, noting that the semantics will never assign both truth and falsity, but sometimes neither.

An atomic formula $R \bar{x}$ is undefined if $a$ is undefined on some variable in $\bar{x}$, in all other cases, truth and falsity are defined in the usual bivalent manner. $\neg \varphi$ is true/false if $\varphi$ is false/true. For a formula based on a tree, we construct a game between two players, $V$ ('verifier') and $F$ ('falsifier'), defining the formula to be true if and only if $V$ has a winning strategy and false if and only if $F$ has a winning strategy. We define this game as follows:

Let $\varphi=\langle T, \leq, g\rangle$ be a formula of $\mathcal{N}_{\infty}, \mathfrak{A}$ a model and $a$ an assignment function $\varphi$. Plays of the game determined by these three items consist of a (possibly transfinite) sequence of stages. Each such stage is given by a node of the tree (i.e., an element of $T$ ) and an assignment function. $V$ wins the game if the node of the last stage is labeled by a formula which is true in $\mathfrak{A}$ and the assignment function of the last stage; $F$ wins the game if this formula is false relative to these parameters; the game is a draw otherwise. Note that the game can be a draw because the formula labeling the node of the last stage is neither true nor false relative to the relevant parameters, or because there is no last stage. We define the plays of the game determined by $\varphi, \mathfrak{A}$ and $a$ by transfinite induction (where the play concludes once the node of the current stage is a leaf of the tree):

- Stage 0 is given by the root node of $\varphi$ and $a$.

- If stage $\alpha$ is given by node $t$ and assignment $b$, then:

- If $t$ is labeled by $\vee$, then $V$ chooses one of its successors $t^{\prime}$; stage $n+1$ is given by $t^{\prime}$ and $b$.

- If $t$ is labeled by $\wedge$, then $F$ chooses one of its successors $t^{\prime}$; stage $n+1$ is given by $t^{\prime}$ and $b$.

- If $t$ is labeled by $\exists x$, then $V$ chooses an element $o$ of the domain of $\mathfrak{A}$; stage $n+1$ is given by the successor of $t$ and $b[o / x]$.

- If $t$ is labeled by $\forall x$, then $F$ chooses an element $o$ of the domain of $\mathfrak{A}$; stage $n+1$ is given by the successor of $t$ and $b[o / x]$.

- Stage $\lambda$, for limit ordinal $\lambda$, is the limit of the stages $<\lambda$, defined as follows: Let $\left\langle t_{\alpha}: \alpha<\lambda\right\rangle$ and $\left\langle b_{\alpha}: \alpha<\lambda\right\rangle$ be the sequences of nodes and assignment functions of the stages $<\lambda$. Then we define the node of the limit stage to be the first node after the elements of $\left\langle t_{\alpha}: \alpha<\lambda\right\rangle$ (this is guaranteed to be unique by constraint (c) of the definition of trees above). 
We define the assignment of the limit stage to map every variable $x$ to the element $o$ such that for some $\alpha<\lambda, b_{\beta}(x)=o$ for all $\beta<\lambda$ such that $\alpha<\beta$, and to be undefined on $x$ of there is no such element.

This concludes the definition of $\mathcal{N}_{\infty \infty}$. As noted in Rantala (1979, p. 122), we can turn every sentence of $\mathcal{L}_{\infty \infty}$ into an equivalent sentence of $\mathcal{N}_{\infty \infty}$ by replacing every quantification over a set of variables of size $\kappa$ by an infinite sequence of existential quantifiers of length $\kappa$. However, there are sentences of $\mathcal{N}_{\infty}$ which have no equivalent in $\mathcal{L}_{\infty \infty}$; this follows from the fact that the so-called game quantifier, which can be thought of as an $\omega$ sequence of alternating existential and universal quantifiers, is clearly definable in $\mathcal{N}_{\infty \infty}$, but as noted in Kolaitis (1985, p. 370), it can be shown not to be definable in $\mathcal{L}_{\infty}$ (see Väänänen (2011, p. 244, Proposition 9.38) for a proof).

Formulas of the modal extension $\mathcal{N}_{\infty \infty}^{\mathrm{mq}}$ are defined as in the case of wellfounded languages by adding clauses for the resources (i) - (iv) specified above. More precisely, we define a formula of $\mathcal{N}_{\infty \infty}^{\mathrm{mq}}$ using the following recursion:

- For any relation symbol $R$ and individual variables $\bar{x}, R \bar{x}$ is a formula.

- If $\varphi$ is a formula, then $\neg \varphi$ is a formula.

- $\xi$ is a formula, for any world variable $\xi$.

- $\mathcal{Q} \overline{\bar{x}} \bar{\varphi}$ is a formula, given that $\mathcal{Q}$ is a generalized quantifier, each $\bar{x}_{i}$ is a sequence of variables and $\varphi_{i}$ is a formula.

- $\langle T, \leq, g\rangle$ is a formula, given that

$-\langle T, \leq\rangle$ is a tree (as defined above),

- $g$ is a function on $T$ mapping any leaf to a formula and any non-leaf to $\neg, \vee, \wedge, \exists x, \forall x, \exists \xi, \forall \xi$ or @ $@$ (for some individual variable $x$ and world variable $\xi$ ), and

- if $t$ is not a leaf and $g(t) \notin\{\wedge, \vee\}$, then $t$ has a unique successor.

We define truth and falsity of a formula $\varphi$ of $\mathcal{N}_{\infty \infty}^{\mathrm{mq}}$ in a Kripke model $\mathfrak{M}$ and a world $w$ relative to an assignment function $a$. The conditions for atomic formulas and negation are as above. A world variable $\xi$ is true in $w$ if and only if $w$ is assigned to it; it is false in $w$ if and only if another world is assigned to it; and so it is undefined if the assignment function is not defined on it. Similarly, the truth-conditions for generalized quantifiers are standard, except that $\mathcal{Q} \overline{\bar{x}} \bar{\varphi}$ is undefined if for some $i$ and sequence $\bar{o}$ of elements in $D_{w}, \varphi_{i}$ is undefined in $\mathfrak{M}, w$ and $a\left[\bar{o} / \bar{x}_{i}\right]$. The definition of truth of a tree-based formula differs only in the construction of the relevant game. Given a formula $\varphi=\langle T, \leq, g\rangle$ of $\mathcal{L}_{\infty}^{\mathrm{mq}}$, a Kripke model $\mathfrak{M}$, a world $w$ of $\mathfrak{M}$ and an assignment function $a$, this is defined as follows: A stage of the game is given by an element of $T$, a world (which might be undefined) and an assignment function. Plays are defined as above, with obvious minor amendments, as well as the following new rules:

- Stage 0 is given by the root node of $\varphi, w$ and $a$.

- If stage $\alpha$ is given by node $t$, world $v$ and assignment $b$, then:

- If $t$ is labeled by $\exists x$, then $V$ chooses an element $o \in D_{v}$; stage $n+1$ is given by the successor of $t, v$ and $b[o / x]$. 
- If $t$ is labeled by $\forall x$, then $F$ chooses an element $o \in D_{v}$; stage $n+1$ is given by the successor of $t, v$ and $b[o / x]$.

- If $t$ is labeled by $\exists \xi$, then $V$ chooses a world $u$; stage $n+1$ is given by the successor of $t, v$ and $b[u / \xi]$.

- If $t$ is labeled by $\forall \xi$, then $F$ chooses a world $u$; stage $n+1$ is given by the successor of $t, v$ and $b[u / \xi]$.

- If $t$ is labeled by @ $\xi$, then stage $n+1$ is given by the successor of $t$, $b(\xi)$ and $b$.

- Stage $\lambda$ is given as above, except that the world of the limit stage is the world $v$ such that for some $\alpha<\lambda$, the world of stage $\beta$ is $v$ for all $\beta<\lambda$ such that $\alpha<\beta$, and is undefined if there is no such element.

This concludes the definition of $\mathcal{N}_{\infty \infty}^{\mathrm{mq}}$. As before, we note that every sentence of $\mathcal{L}_{\infty}^{\mathrm{mq}}$ can be turned into an equivalent sentence of $\mathcal{N}_{\infty}^{\mathrm{mq}}$. As in the case of well-founded languages, we use $\vDash$ to express truth of a formula relative to the appropriate parameters, and now use $\neq$ similarly for falsity.

Our unusual way of setting up $\mathcal{N}_{\infty \infty}$ and $\mathcal{N}_{\infty \infty}^{\mathrm{mq}}$ is mainly motivated by the inclusion of all generalized quantifiers in the latter language. While a number of generalized quantifiers can be given a natural game-theoretic semantics (see Pietarinen (2007)), to our knowledge, there is no general game-theoretic semantics for arbitrary generalized quantifiers. (Engström (2012) makes steps in this direction, but his constructions only apply to a limited class of generalized quantifiers and also require us to move from a two-player game to a game between teams of players.) We therefore have to combine a truth-conditional semantics for generalized quantifiers with a game-theoretic semantics for infinitary embeddings; the above hybrid provides a natural and general way of doing so.

Another feature of our presentation to note is the definition of limit stages of a game, which explicitly allows for variables not to be assigned an element this can happen if there is an infinite sequence of quantifiers binding the same variables and the players keep choosing different elements for it. Such cases are usually implicitly assumed to be ruled out syntactically; Oikkonen (1979, p. 104, (iv)) does so explicitly. Ruling out these cases syntactically is unnatural in the setting of $\mathcal{N}_{\infty \infty}^{\mathrm{mq}}$ since the same problem occurs in the case of the world of evaluation for formulas with branches containing an infinite sequence of $\nabla_{s}-$ of course, the formulas we are most interested in are exactly such formulas, so we cannot rule these out syntactically. Adapting this treatment, one could also allow for infinite nestings of negations, but since this would make no difference to the following results, it is omitted for simplicity.

\section{A.4 Definability in non-well-founded languages}

To characterize which generalized quantifiers are modalizable in $\mathcal{N}_{\infty}^{\mathrm{mq}}$, we adapt a number of ideas from Williamson (2010, Appendix 3). We start with some definitions, observations and lemmas. Define a function ${ }^{n}$ for every natural number $n$ which maps every model $\mathfrak{A}$ to the Kripke model $\mathfrak{A}^{n}=\langle W, D, i, \emptyset\rangle$, where $W$ is the set of subsets of $|\mathfrak{A}|$ of cardinality $\leq n, D_{w}=w$ for all $w \in W$, and $i(R)(w)=R^{\mathfrak{A}} \cap w^{n}$ for all relation symbols $R$. 
Note that for every generalized quantifier $\mathcal{Q}$ and $n<\omega$, there is a formula $\delta_{\mathcal{Q}}^{n}$ of $\mathcal{L}_{\omega \omega}$ which defines $\mathcal{Q}$ on models up to cardinality $n$; i.e., for every model $\mathfrak{A}$ of cardinality $\leq n, \mathfrak{A} \vDash \delta_{\mathcal{Q}}^{n}$ iff $\mathfrak{A} \vDash \gamma_{\mathcal{Q}}$. We write $\delta_{\mathcal{Q}}^{n}(\bar{\varphi})$ for the result of replacing the atomic formulas in $\delta_{\mathcal{Q}}^{n}$ by the formulas of $\bar{\varphi}$, leaving the appropriate replacements of variables implicit. Define the relativization of a generalized quantifier $Q$ of type $\bar{n}$ to be the generalized quantifier $Q^{\text {rel }}$ of type $\langle 1, \bar{n}\rangle$ such that for all sets $D$ and $D^{\prime} \subseteq D$ and sequence of relations $\bar{R}$ on $D, Q_{D}^{\text {rel }}\left(D^{\prime}, \bar{R}\right)$ if and only if $Q_{D}^{\prime}\left(\bar{R}^{\prime}\right)$, where $\bar{R}^{\prime}$ is the sequence of relations in $\bar{R}$ restricted to $D^{\prime}$. See Westerståhl (2011, section 7 ) for a more precise definition.

For every $n<\omega$, we define a mapping $[\cdot]_{n}$ from $\mathcal{N}_{\infty \infty}^{\mathrm{mq}}$ to $\mathcal{N}_{\infty \infty}$ by a number of replacements. For present purposes, we assume that all individual variables of $\mathcal{N}_{\infty \infty}$ are individual variables of $\mathcal{N}_{\infty \infty}^{\mathrm{mq}}$, and that in addition, $\mathcal{N}_{\infty \infty}$ contains distinct individual variables $\xi_{0}, \ldots, \xi_{n-1}$ for each world variable $\xi$ of $\mathcal{N}_{\infty}^{\mathrm{mq}}$, as well as new individual variables $w_{0}, \ldots, w_{n-1}$. We replace

- $R \bar{x}$ by $R \bar{x} \wedge \bigwedge_{x \in \bar{x}} \bigvee_{i<n} x=w_{i}$

- $\xi$ by $\forall x\left(\bigvee_{i<n} x=\xi_{i} \leftrightarrow \bigvee_{i<n} x=w_{i}\right)$

- $\mathcal{Q} \overline{\bar{x}} \bar{\varphi}$ by $\delta_{\mathcal{Q}^{\text {rel }}}^{n}\left(\bigvee_{i<n} x=w_{i}, \bar{\varphi}\right)$, where $x$ is not free in any $\bar{\varphi}$

- $\exists x \varphi$ by $\exists x\left(\bigvee_{i<n} x=w_{i} \wedge \varphi\right)$

- $\forall x \varphi$ by $\forall x\left(\bigvee_{i<n} x=w_{i} \rightarrow \varphi\right)$

- $\exists \xi \varphi$ by $\exists \bar{\xi} \varphi$

- $\forall \xi \varphi$ by $\forall \bar{\xi} \varphi$

- $@ \xi \varphi$ by $\forall \bar{w}\left(\bigwedge_{i<n} \xi_{i}=w_{i} \rightarrow \varphi\right)$

For any sentence sentence $\varphi$ of $\mathcal{N}_{\infty}^{\mathrm{mq}}$, we define $[\varphi]_{n}$ to be $\forall \bar{w} \varphi^{\prime}$, where $\varphi^{\prime}$ is the result of carrying out the above replacements on $\varphi$. Note that we write $\mathfrak{M} \vDash \varphi / \mathfrak{M}=\varphi$ for $\varphi$ being true/false in every world of the Kripke model $\mathfrak{M}$.

Lemma 3. For any $n<\omega$, sentence $\varphi$ of $\mathcal{N}_{\infty}^{\mathrm{mq}}$ and model $\mathfrak{A}$, if all relation symbols occurring in $\varphi$ are of arity $\leq n$, then $\mathfrak{A}^{n} \vDash \varphi$ iff $\mathfrak{A} \vDash[\varphi]_{n}$, and $\mathfrak{A}^{n}=\varphi$ iff $\mathfrak{A}=[\varphi]_{n}$.

Proof. By induction on the complexity of $\varphi$.

Lemma 4. Let $\mathfrak{A}$ be a model and $\mathcal{Q}$ a generalized quantifier of type $\bar{n}$. Then $\mathfrak{A} \vDash \gamma_{\mathcal{Q}}$ iff $\mathfrak{A}^{\max (\bar{n})} \vDash \gamma_{\mathcal{Q}^{\circ}}$, and $\mathfrak{A}=\gamma_{\mathcal{Q}}$ iff $\mathfrak{A}^{\max (\bar{n})}=\gamma_{\mathcal{Q}^{o}}$.

Proof. By the construction of $\mathfrak{A}^{\max (\bar{n})}$.

Theorem 5. The modalization of a generalized quantifier $\mathcal{Q}$ is definable in $\mathcal{N}_{\infty \infty}^{\mathrm{mq}}$ if and only if $\mathcal{Q}$ is definable in $\mathcal{N}_{\infty \infty}$.

Proof. The right-to-left direction can be established as in the proof of Theorem 2. For the left-to-right direction, let $\mathcal{Q}$ be a generalized quantifier of type $\bar{n}$ whose modalization $\mathcal{Q}^{O}$ is definable in $\mathcal{N}_{\infty}^{\mathrm{mq}}$. Then there is a formula $\varphi$ of $\mathcal{N}_{\infty \infty}^{\mathrm{mq}}$ such that for all models $\mathfrak{A}, \mathfrak{A}^{\max (\bar{n})} \vDash \gamma_{\mathcal{Q}^{O}}$ iff $\mathfrak{A}^{\max (\bar{n})} \vDash \varphi$, and $\mathfrak{A}^{\max (\bar{n})} \neq \gamma_{\mathcal{Q}^{O}}$ iff $\mathfrak{A}^{\max (\bar{n})} \neq \varphi$. By Lemmas 3 and 4 , it follows that for all models $\mathfrak{A}, \mathfrak{A} \vDash \gamma_{\mathcal{Q}}$ iff $\mathfrak{A} \vDash[\varphi]_{\max (\bar{n})}$, and $\mathfrak{A} \neq \gamma_{\mathcal{Q}}$ iff $\mathfrak{A} \neq[\varphi]_{\max (\bar{n})}$. So $\mathcal{Q}$ is definable in $\mathcal{N}_{\infty \infty}$. 


\section{A.5 Conclusions and remarks}

Using Theorems 1,2 and 5, we can use standard results on the undefinability of generalized quantifiers in $\mathcal{L}_{\omega \omega}, \mathcal{L}_{\infty \omega}$ and $\mathcal{N}_{\infty \infty}$ to deduce the undefinability of the corresponding modalized generalized quantifiers in $\mathcal{L}_{\omega \omega}^{\mathrm{mq}}, \mathcal{L}_{\infty \infty}^{\mathrm{mq}}$ and $\mathcal{N}_{\infty \infty}^{\mathrm{mq}}$. In particular, we note the following, using open English sentences to denote the relevant generalized quantifiers:

- 'there are infinitely many ...' is not definable in $\mathcal{L}_{\omega \omega}$.

- 'there are uncountably many ...' is not definable in $\mathcal{L}_{\infty}$.

- 'most ... are ...' is not definable in $\mathcal{N}_{\infty \infty}$.

The first follows from the compactness of $\mathcal{L}_{\omega \omega}$, the second from Fact 1.1.1 of Dickmann (1985, p. 318), and the last from a version of the downward Löwenheim-Skolem theorem for $\mathcal{N}_{\infty \infty}$ (Karttunen, 1983, p. 228, Theorem 2.1).

Let us also add a few remarks on the robustness of the above results: First, we impose a negative free logic in the model theory for our modal languages, requiring the interpretation of a relation at a world in a Kripke model to be restricted to the domain of that world. All of the results presented above go through as well if this assumption is dropped. Second, we used the relatively rich resources of world quantification rather than the more modest generalized actuality operators used in Williamson (2013, chapter 7 ). Since the latter are easily definable using the former, it is clear that our choice does not weaken the direction of our results which deduces the definability of $\mathcal{Q}$ from the definability of $\mathcal{Q}^{O}$ in the relevant languages. And in the mapping which establishes the reverse direction, we could have used the operators Williamson uses, so all of the results presented here are independent of this choice.

Finally, note that the preceding results are established using two distinct proof strategies; the proofs for well-founded languages employ the use of backand-forth systems as in Fritz (2013), while the proofs for non-well-founded languages proceed roughly along the lines of Williamson (2010, Appendix 3). Call the latter the 'direct method' and the former the 'indirect method'. The direct method is applicable to well-founded languages as well, and might even provide simpler proofs. However, the indirect method gives us a way of constructing Kripke models which are equivalent up to some modal depth but disagree on the canonical sentence of a modalized quantifier; this makes it easy to see how the result can be strengthened by enriching the language under consideration. E.g., it is clear from this strategy that the result is not affected by expanding the language to include plural quantifiers. Plural quantifiers can also be accommodated on the direct method, but how to do so is is less obvious. Beside simplicity, the direct method has the advantage of not requiring us to characterize the equivalence of models up to a given quantifier rank in terms of a back-and-forth system. This need not be an obstacle to applying it to $\mathcal{N}_{\infty}$, as back-and-forth systems have been developed for non-well-founded languages; see Rantala (1979) and Karttunen (1979). 